\title{
Latest Advances in Molecular Imaging Instrumentation
}

Bernd J. Pichler, Hans F. Wehrl, and Martin S. Judenhofer

Laboratory for Preclinical Imaging and Imaging Technology of the Werner Siemens Foundation, Department of Radiology, Eberhard Karls University Tübingen, Tübingen, Germany

This review concentrates on the latest advances in molecular imaging technology, including PET, MRI, and optical imaging. In PET, significant improvements in tumor detection and image resolution have been achieved by introducing new scintillation materials, iterative image reconstruction, and correction methods. These advances enabled the first clinical scanners capable of time-of-flight detection and incorporating point-spread-function reconstruction to compensate for depth-of-interaction effects. In the field of MRI, the most important developments in recent years have mainly been MRI systems with higher field strengths and improved radiofrequency coil technology. Hyperpolarized imaging, functional MRI, and MR spectroscopy provide molecular information in vivo. A special focus of this review article is multimodality imaging and, in particular, the emerging field of combined PET/MRI.

Key Words: instrumentation; PET; PET/MRI; MRI; optical imaging

J Nucl Med 2008; 49:5S-23S

DOI: $10.2967 /$ jnumed.108.045880

$\mathbf{P}$

ET is a high-performance imaging technology that can image the whole-body distribution of positron-emitting biomarkers with high sensitivity. The invention of PET dates back more than $30 \mathrm{y}$. However, the acceptance of PET as a routine clinical diagnostic tool has been hampered by the need for an on-site cyclotron and a radiochemistry unit for the production of the short-lived radiotracers (1-7). With the availability of commercial radiopharmacies that supply PET probes and with the relatively broad insurance coverage for a variety of clinical indications, the number of PET centers now totals more than 2,000 worldwide.

Major technologic milestones in the development of PET include the discovery of faster and brighter scintillators such as lutetium oxyorthosilicate (LSO), gadolinium oxyorthosilicate, and lutetium yttrium oxyorthosilicate. Furthermore, the continuing development of the detectors, progressing

\footnotetext{
Received Mar. 28, 2008; revision accepted Apr. 30, 2008.

For correspondence or reprints contact: Bernd J. Pichler, Laboratory for Preclinical Imaging and Imaging Technology of the Werner SiemensFoundation, Department of Radiology, Eberhard Karls University Tübingen, Röntgenweg 13, 72076 Tübingen, Germany.

E-mail: Bernd.Pichler@med.uni-tuebingen.de

COPYRIGHT @ 2008 by the Society of Nuclear Medicine, Inc.
}

from one-to-one scintillator-to-photomultiplier-tube (PMT) coupling $(8,9)$ to advanced block and Anger readout schemes, helps to limit the costs of a PET scanner by providing a multiplexed readout and reduced electronic channels (10-13).

The use of faster scintillators enabled the transition from 2-dimensional data acquisitions for whole-body PET, using lead or tungsten collimators as septa (14-16), to 3-dimensional (3D) acquisitions (17). However, approximately an 8-fold increase in detection sensitivity is accompanied by an increased fraction of random and scattered photon events leading to degradation of image quality if not corrected during image reconstruction (18). This degradation effectively reduces the increase in sensitivity from a factor of 8 to approximately 5-fold. To achieve high-quality and quantitative PET data, various image reconstruction techniques have evolved (19). One simple approach is filtered backprojection, which is computationally fast but results in poor image quality if the count statistics of the available PET data are inadequate. Better image quality and improved spatial resolution are achieved by iterative methods such as ordered-subset expectation maximization or maximumlikelihood expectation maximization algorithms (20) together with a posteriori information. Iterative algorithms use the underlying physical model of the scanner to implement geometric factors and corrections for photon scatter and detector penetration (21-23). However, iterative reconstruction approaches are much more computationally intense than filtered backprojection and do not necessarily converge. Appropriate corrections for dead time, detector normalization, photon scatter, random events, and in particular photon attenuation have been developed to obtain quantitative image data.

Through the integration of these software and hardware advances, PET has matured as a technology, providing whole-body patient scans within 10-20 min with a spatial resolution of 4-7 $\mathrm{mm}$ in the reconstructed image. Because of its high sensitivity, the large variety of available probes, and the potential for development of new biomarkers, PET is a powerful tool for clinical diagnosis and biomedical research. An important milestone in PET has been the introduction of multimodality imaging —in particular, combined 
PET/CT - to provide functional and morphologic information in a single patient scan $(24,25)$.

Although PET has invigorated nuclear medicine by initiating the field of clinical molecular imaging, MRI has also had a profound effect on the practice of radiology. The wide variety of imaging sequences, along with better softtissue contrast than that of $\mathrm{CT}$, makes MRI an important diagnostic tool in the areas of cardiology, neurology, and oncology. In addition to the anatomic information delivered by MRI, MR spectroscopy provides spectral and therefore molecular information about tissue composition in vivo. More recently, 7- or even 9.4-T human scanners have been introduced that exhibit superb spatial resolution and a high signal-to-noise ratio (SNR). These tomographs are especially of interest for studying the physiology of the human brain using functional MRI (fMRI) to visualize and quantify blood-oxygenation-level dependency (BOLD). Another important step was the improvement in radiofrequency coil technology; in this regard, at least for the preclinical field, cryo-coils have entered the market. A current focus of MRI research concentrates on functional imaging using hyperpolarized substances such as ${ }^{13} \mathrm{C}$ or ${ }^{19} \mathrm{~F}$.

In vivo molecular imaging tools include PET, MRI, SPECT, and optical imaging. MRI, PET, and SPECT have demonstrated great clinical value and utility, whereas clinical applications of optical imaging are still in their infancy. All modalities play an important role in preclinical research, but there are some fundamental technical differences between clinical and preclinical imaging. Light does not propagate deeply in tissues, and therefore although whole-body imaging with optical technologies is possible for mice, it is not possible for humans. Therefore, throughout this review we will discuss each modality in the context of both preclinical and clinical applications. However, optical imaging in general, and PET and MRI in particular, have undergone the most significant technical advancements over the last few years, and we have therefore elected to focus primarily on these 2 modalities. In addition, we briefly describe the principles and advances in optical imaging and CT.

\section{PET}

\section{Advances in PET Detector Technology}

Important steps to improve PET performance have been driven by advances in $\gamma$-detector technology. The first PET images were acquired in the 1970s by using a hexagonal arrangement of 24 single sodium iodide (NaI) scintillation crystals, individually coupled to PMTs (Fig. 1). Although $\mathrm{NaI}$ is a nearly ideal scintillator material for detecting lowenergy $\gamma$-photons in SPECT cameras, the probability of interaction is low for $511-\mathrm{keV} \gamma$-photons, reducing the sensitivity of the PET system significantly. In addition, compared with LSO, the relatively slow scintillation decay time of $230 \mathrm{~ns}$ for $\mathrm{NaI}$ precludes fast coincidence timing, which is desired in PET applications to reduce data contamination by random events. Nevertheless, the first PET images were successfully acquired with this setup (Fig. 1C). Significant improvements in the spatial resolution of PET images were driven not only by a higher granularity of the scintillation blocks but also by new scintillator materials yielding more light per mega-electron volt, shorter scintillation decay times, and higher stopping power. Although bismuth germanate was for many years the crystal material of choice for PET, the advent of LSO (doped with cerium) in the late 1990s provided a scintillator with a higher light yield and approximately 7 times better timing performance (Table 1) (26). Thus, LSO and its derivates such as lutetium yttrium oxyorthosilicate (doped with cerium) paved the way for fast coincidence timing thereby reducing random events and improving image quality. However, a drawback of LSO is its intrinsic background radioactivity due to the ${ }^{176} \mathrm{Lu}$, which can increase the singles counting rate. Although a one-to-one coupling of scintillator and light detector would be advantageous to improve the counting rate performance, energy, and timing resolution of PET $(21,27,28)$, clinical
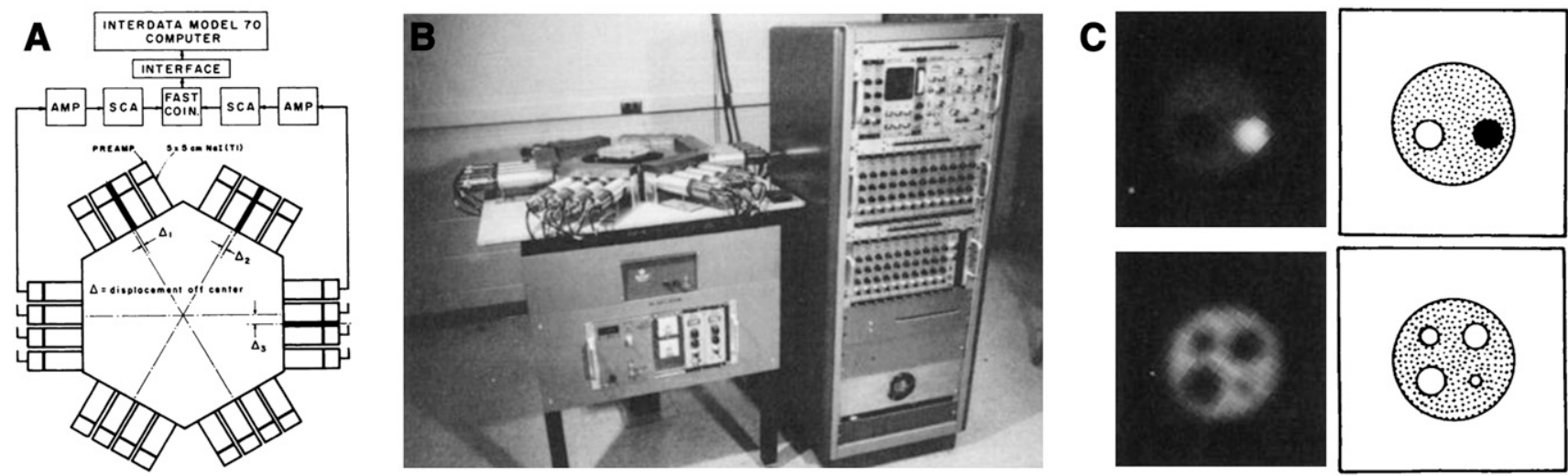

FIGURE 1. First prototype PET scanner (positron emission transaxial tomograph, PETT) (1). (A and B) Schematic and picture show that total of 24 crystals were arranged in hexagonal shape providing 1 transaxial image slice. (C) Image of phantom filled with activity and cold spots that could be imaged with this apparatus. (Reprinted from (171).) 
TABLE 1

Scintillation Materials Used in Nuclear Medicine Instrumentation (168-170)

\begin{tabular}{|c|c|c|c|c|c|c|c|c|}
\hline Property & $\operatorname{Nal}(\mathrm{TI})$ & BGO & LSO & YSO & GSY & $\mathrm{BaF}$ & $\mathrm{LaBr}_{3}$ & LYSO \\
\hline Density $\left(\mathrm{g} / \mathrm{cm}^{3}\right)$ & 3.67 & 7.13 & 7.4 & 4.53 & 6.71 & 4.89 & 5.3 & 5.31 \\
\hline Effective Z & 50.6 & 74.2 & 65.5 & 34.2 & 58.6 & 52.2 & - & 54 \\
\hline Attenuation length & 2.88 & 1.05 & 1.16 & 2.58 & 1.43 & 2.2 & 2.1 & 2.0 \\
\hline Decay constant (ns) & 230 & 300 & 40 & 70 & 60 & 0.6 & 15 & 53 \\
\hline Relative light output (\%) & 100 & 15 & 75 & 118 & 25 & 5 & 160 & 76 \\
\hline Wavelength $(\lambda[\mathrm{nm}])$ & 410 & 480 & 420 & 420 & 440 & 220 & 360 & 420 \\
\hline Index of refraction & 1.85 & 2.15 & 1.82 & 1.8 & 1.91 & 1.56 & 1.9 & 1.81 \\
\hline Hygroscopic? & Yes & No & No & No & No & No & Yes & No \\
\hline
\end{tabular}

BGO = bismuth germanate; GSO = gadolinium oxyorthosilicate; LYSO = lutetium yttrium oxyorthosilicate; YSO = yttrium oxyorthosilicate.

PET scanners use a light-sharing readout that reduces the number of electronic channels, which in turn reduces PET production costs. Today's PET detector arrangements are still based on Anger readout $(10,29-32)$ or on a block detector principle (Fig. 2). Both methods use light sharing and map many small scintillation crystals to few light detectors, in most cases PMTs. This approach results in a multiplexing factor of 16 or even higher (30-32). Here as well, the latest generation of scintillators is advantageous for improving block detector performance; brighter scintillators allow for an increase in the multiplexing factor and also for finer crystal segmentation (Fig. 3).

There is an ongoing discussion about the ideal crystal size. The smaller the individual crystal of a PET detector, the higher is the spatial resolution; however, smaller crystals cause the number of lines of response (LORs) in a scanner to increase, potentially leading to a statistical problem for the reconstruction if the scan time is short and the injected radioactivity remains low. However, in theory, a binning of single crystal pixels, such as those in CCD cameras, can improve the statistics per LOR at the cost of spatial resolution. A main factor limiting clinical systems from achieving higher resolution is photon noncolinearity; thus, in clinical PET scanners the pixel cross-section is still between $4 \times 4$ and $6.5 \times 6.5 \mathrm{~mm}(30-32)$, whereas small-animal systems, which require a higher resolution but have a smaller field of view in the axial and transaxial directions and thus fewer LORs, have pixels about $1.5 \times 1.5 \mathrm{~mm}$ in cross section (Fig. 3C). Feasibility studies focusing on small crystals for PET detectors have shown that, in theory, the smallest achievable resolution is on the order of $0.4 \mathrm{~mm}$ in the reconstructed image (33). However, resolutions beyond that are unlikely because the intrinsic physical limitations, such as positron range and noncolinearity of the annihilation $\gamma$-photons, will dominate. Detectors based on such fine pixelated crystal blocks (Fig. 4) (34) might find their way into small-animal PET or high-resolution organ-specific dedicated human scanners for breast or brain imaging but will probably not enter standard clinical systems because of the significant costs for readout and manufacturing.
The requisite for a PET detector is to provide not only good spatial resolution but also high sensitivity that minimizes both patient scan time and the injected radioactivity. The sensitivity of a PET detector can be increased by using crystals with a higher stopping power, maximizing the packing fraction, and increasing the length. Thus, in the design of a PET scanner, sensitivity and spatial resolution are often a compromise. In this respect, detector researchers are studying ways of making crystals deeper to increase the overall PET sensitivity without affecting spatial resolution. One possibility for achieving this is by detecting the depth of interaction (DOI), or the location within the crystal where the photon is absorbed. There are several potential ways of generating a DOI detector, although all these approaches are experimental and have not entered standard commercial products yet. The only exceptions are animal PET systems such as the eXplore VISTA (GE Healthcare) (35) and the LabPET (Gamma Medica) (36), as well as the high-resolution brain scanner HRRT (37) (Siemens Medical Solutions), which uses 2 layers of different crystal types stacked together-the phoswich design (Fig. 5A). In this concept, the DOI is determined electronically by analyzing the scintillation decay time of the different crystal types. Thus, the DOI is based on the interaction of the photons in the front or back crystal layer. With this approach, the number of light detectors and electronic channels does not increase. More advanced systems, which are still experimental, concentrate on resolving a continuous DOI within the entire crystal length (Fig. 5B) (38). The DOI is of particular importance for high-resolution PET scanners with a small-diameter bore, such as dedicated brain scanners or animal PET systems $(11,27,39)$.

Although most commercial clinical and small-animal PET systems still use PMTs as light detectors, a large fraction of PET research focuses on replacing PMTs by novel semiconductor-based light detectors. The reason for still using PMTs in PET although they are bulky and relatively expensive is that they are reliable in operation and so far provide the best performance with respect to electronic noise and timing properties, when compared with 


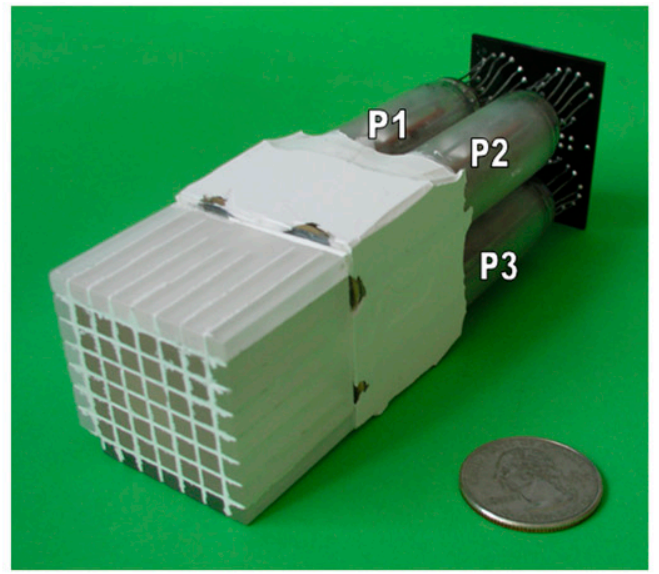

B

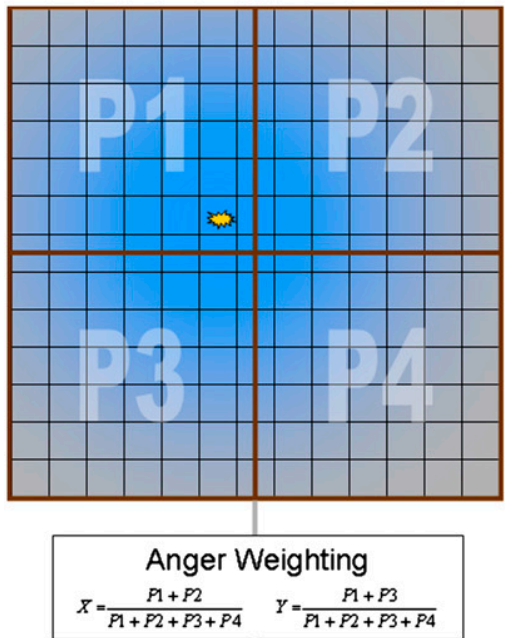

C

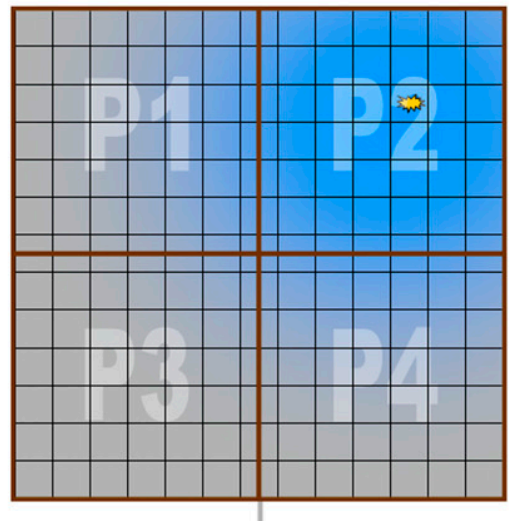

\section{D}

FIGURE 2. Block detector. (A) Example of PMT-bismuth germanate block detector from clinical PET scanner. Readout is performed using only the 4 PMTs that are connected to pixilated scintillator block. Light sharing is used to distribute light originating from single pixel between the 4 readout PMTs ( $P 1$, P2, P3, P4). (B and C) Depending on crystal position, light will be uniquely distributed to readout PMTs. (D) Using Anger weighting algorithm on measured signals, position of incident event can be calculated and assigned to position profile. (Courtesy of University of Tübingen.)

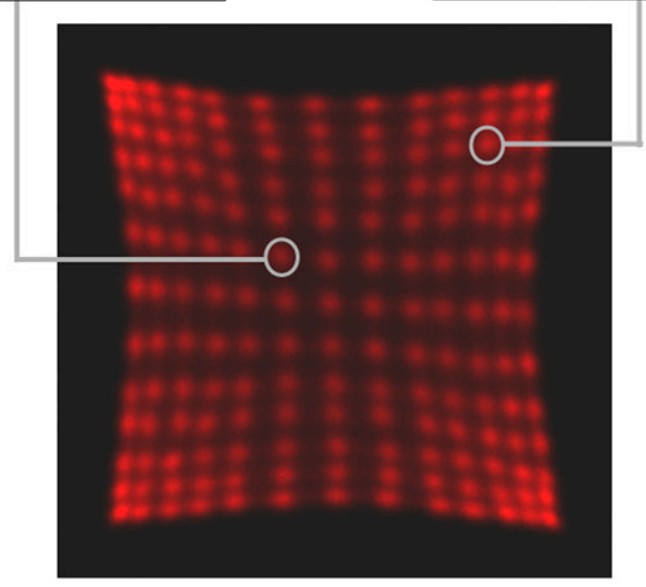

all other light detectors. Avalanche photodiodes (APDs) have been the subject of intensive research for the last $15 \mathrm{y}$ $(3-5,40)$. The first evaluations of APDs in prototype smallanimal PET scanners proved their feasibility as light detectors. Major advantages of APDs are their compactness, high quantum efficiency that consequently provides an energy resolution close to that of PMTs, and low bias voltage, which is a helpful feature for an inexpensive system design
(Fig. 6). Over the years, the reliability and robustness of APDs have improved significantly, and now they operate for many years without performance degradation. Most important, APDs are semiconductor devices and therefore have the potential to become less expensive as the production volume increases.

APDs not only are compact but also are insensitive to magnetic fields (41) and therefore ideal as PET light 

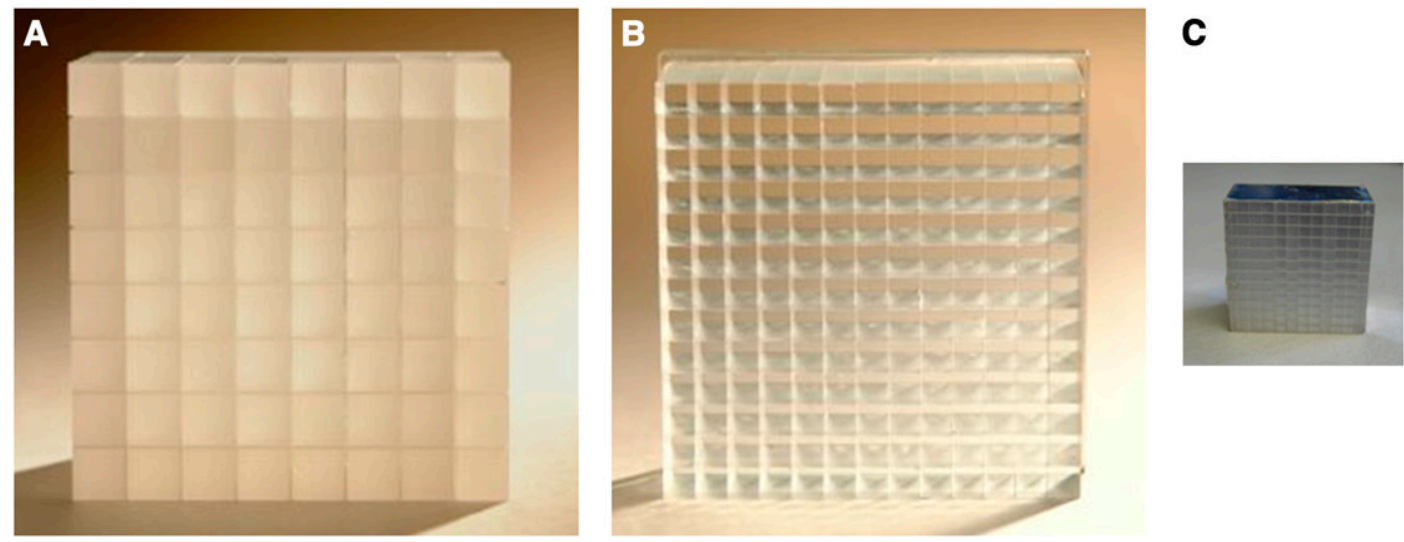

D

FIGURE 3. Scintillation crystal blocks for PET detectors. Size of each individual scintillator used for detector block depends on application field and defines intrinsic resolution of PET scanner. (A) Early clinical PET scanners used $6 \times 6 \mathrm{~mm}$ bismuth germanate crystals arranged in $8 \times 8$ matrix. (B) Newer generations of PET systems (124) use, for instance, $13 \times 13$ LSO array with only $4 \times 4$ $\mathrm{mm}$ cross-sectional crystals. (C) Preclinical systems aim for spatial resolution below $1.5 \mathrm{~mm}$ and use, for example, $12 \times 12$ array of LSO scintillators with size of $1.5 \times 1.5 \times 10 \mathrm{~mm}$. (D) New research has focused on reducing crystal pixel size to improve spatial resolution. Crystal block with crystals of only $0.43 \times 0.43 \mathrm{~mm}$ in cross section was successfully fabricated and read out by Stickel et al. (34). (Panels A and B courtesy of Siemens Medical Solutions; panel C courtesy of University of Tübingen; panel D reprinted from (8).)

detectors in combined PET/MRI scanners (42-45). For an integrated combination of PET and MRI, the PMT cannot be used because its function is based on electron acceleration within a high electric field in a relatively large vacuum tube. Thus, even small magnetic fields can deflect electrons from their original trajectories, leading to a loss of gain.

An interesting alternative to APDs emerged in the early 2000s, when a new generation of Geiger-mode APDs, or so-called siliconPMs (siPMs), was introduced (46). Standard APDs are based on different silicon layers in which an internal gain is built up at the p-n junction by a high electric field, accelerating free electrons, which are generated by scintillation light impacting the APD surface. The p-n junction is only several micrometers thick and therefore makes an APD a compact device. This type of APD is insensitive to magnetic fields because the free path of electrons is short; therefore, the Lorenz force has essentially little chance of deflecting them from their original path. However, APDs are more difficult to manufacture than standard semiconductors and have an increased excess noise when the active area gets larger. Also, standard APDs have a limited electronic gain of approximately $100-1,000$, which is a factor of 1,000-10,000 lower than the gain of
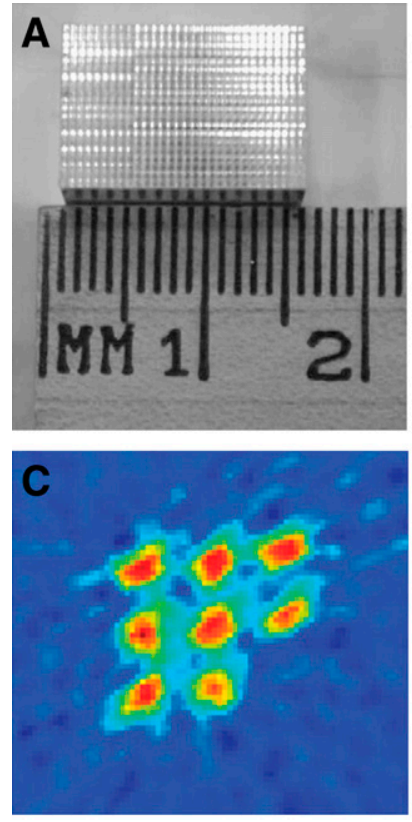

FIGURE 4. Small pixilated block detectors (8). (A) Ambition to achieve smaller resolution encouraged fabrication of LSO crystal blocks with $20 \times 30$ elements, each with cross-section of only $0.43 \times$ $0.43 \mathrm{~mm}$. (B) Even though crystal size is small, position profiles of block could be acquired using 64-channel positionsensitive PMT. (C and D) Two of these LSO blocks have been operated in coincidence to acquire phantom images of $0.5-\mathrm{mm}$ rod source phantom (C) and mouse paw (D). (Reprinted from (8).) 
FIGURE 5. DOI schemes for PET block detectors. Different methods can be used to acquire information on location of $\gamma$-interaction in crystal. (A) One approach is a stack of 2 or more crystal layers with different scintillation properties, which allow for upper and lower crystal layer to be distinguished by discriminating different scintillation decay times. (B) Continuous DOI can be gained by reading scintillation light from both sides of scintillation crystal. In this approach, position-sensitive APDs (PSAPDs) are used to read scintillation light on each side. Comparing amount of light collected on each side of scintillator allows for calculation of DOI with resolution of 3-4 mm (26). All these approaches are challenging technologies and currently not implemented in any standard clinical PET system. (Panel A (C) 1998 IEEE and reprinted with permission of (172); panel B reprinted from (38).)

PMTs and therefore requires dedicated integrating preamplifiers. The lower gain along with the required preamplifiers results in a lower SNR and poorer timing performance for the PET detector. In sharp contrast to standard APDs, the active surface of siPMs is subdivided into small cells several micrometers in size. Each of these cells is operated in Geiger mode; this means essentially that the voltage exceeds the breakdown value at the $p$-n junction and in principle leads to an infinite gain. To prevent destruction of the siPM by heat, a small integrated resistor limits the maximum current. When a photon impacts a cell of the siPM, the probability is high that an avalanche process is initiated, providing an output amplitude of the electrical signal that is proportional to the number of cells fired. The gain achieved with siPMs can exceed $10^{6}$. Because siPMs work in Geiger mode, each cell that detects light provides a signal amplitude independent of the number of photons detected per cell. Thus, to maintain the overall linearity of the siPM output signal, the number of cells needs to be much higher than the number of expected light photons impacting the siPM surface. In essence, siPMs provide high signal amplitudes at the output and can therefore be operated with simple preamplifiers. They are as compact as APDs and insensitive to magnetic fields. Their production is based on a standard CMOS process and thus has the potential for inexpensive mass production. A few years from now, when Geiger-mode APDs have been further researched and optimized, siPMs might drastically change current PET detector technology. They will most likely be the technology of choice for combined PET/MRI scanners and potentially also for combined PET/CT, serving as a dual detector for PET photons and CT x-rays.

In summary, the development of novel, high-sensitivity, and high-resolution PET detectors was a major step improving PET image quality with the potential ability to resolve small lesions in vivo. At the same time, immensely improved computer power, faster scintillators, and advanced electronics have helped to reduce the scanning time and the injected radioactivity dose. This in turn has made time-of-flight (TOF) PET feasible. Thus, the field of PET detector research was, and still is, a key factor for advancing clinical and preclinical PET.

\section{Advances in PET Reconstruction Algorithms}

Iterative reconstruction algorithms have replaced analysisbased filtered backprojection in most clinical and preclinical PET applications. Iterative reconstruction techniques such as maximum-likelihood expectation maximation $(20,47)$, ordered-subset expectation maximization, or maximum a posteriori (48) reconstruction have the advantage of being

FIGURE 6. Example of APD implemented as single detector with $5 \times 5 \mathrm{~mm}$ (center) active surface and monolithic array of 9 APDs each having $5 \times 5 \mathrm{~mm}$ active surface. Thickness of APD is only $1.5 \mathrm{~mm}$, including housing. (Courtesy of University of Tübingen.)

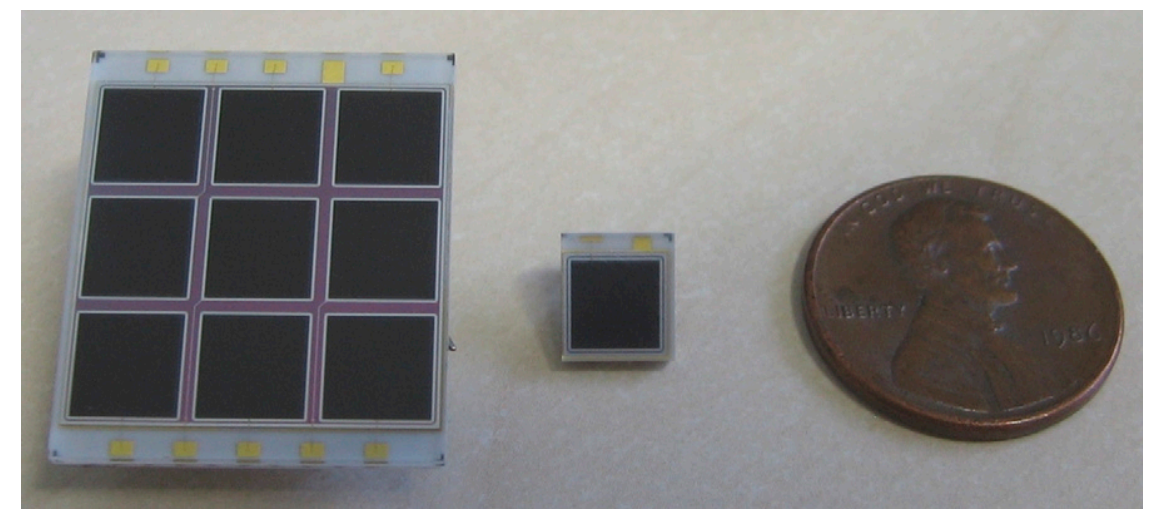


able to include directly in the algorithm the detector-specific parameters of the system and models for attenuation, randoms, and scatter. The result is reduced noise and streak artifacts, well known in filtered backprojection images. In addition, iterative reconstruction is preferable on 3D PET data, especially for whole-body applications. A comprehensive review of iterative reconstruction methods can be found by Leahy and Byrne (49).

A PET detector design will always be a compromise between spatial resolution, sensitivity, and price. A major problem is that deep crystals improve sensitivity but worsen spatial resolution, especially at the edge of the field of view, when no DOI information is provided and the point spread function broadens with oblique penetration (Fig. 7). DOI can be revealed by using a more complex detector design or by including the point spread function into the reconstruction algorithm $(22,23)$. This approach is currently being pursued by at least one manufacturer of commercial PET systems by interpolating between sinogram bins with spatially variant point spread functions for improving the resolution uniformly over the entire field of view and might help to lower the noise in the reconstructed PET images, ultimately leading to higher contrast. As for TOF PET systems, this effect is more predominant for larger patients (Fig. 8), in whom the counting rate is lower because of attenuation and the ratio between scattered and true events is unfavorable.

\section{TOF PET}

In conventional PET, once a photon from a positron electron annihilation event is detected, the preset coinci-

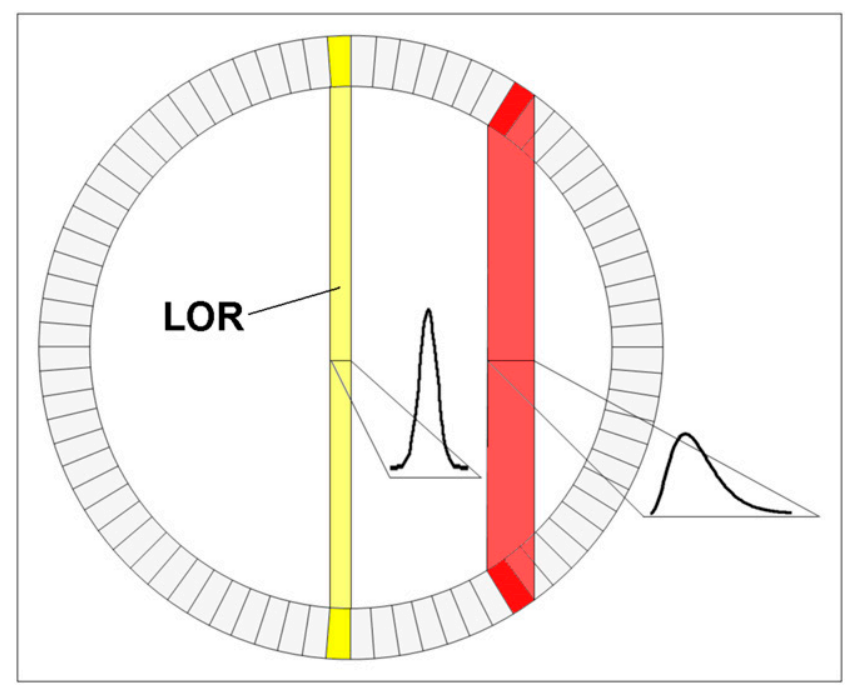

FIGURE 7. To achieve high sensitivity in PET system, scintillators cannot be very short because of resulting limited stopping probability for $511-\mathrm{keV} \gamma$-quanta. If longer crystal is used and no DOI information is available, image resolution will gradually degrade when going toward edge of field of view because LOR starts to broaden. Therefore, one has to compromise between sensitivity (long crystal) and image quality. (Courtesy of University of Tübingen.) dence timing window of the scanner allows a certain time in order for detection of a second coincident photon to be considered as originating from the same annihilation. This timing window is on the order of about $10 \mathrm{~ns}$ in standard PET scanners. Therefore, considering that the speed of light is $2.99 \times 10^{8} \mathrm{~m} / \mathrm{s}$, this window does not allow for exact determination of the annihilation position. In fact, the only spatial information is derived from the position of the LOR between the 2 opposing detectors (Fig. 9). The idea of TOF PET is basically to increase the SNR of the PET images by including more accurate information about the location of the annihilation event. TOF PET is not a new concept, having been investigated in the 1980s but with moderate success. Because the scintillators investigated at that time $\left(\mathrm{NaI}, \mathrm{CsF}, \mathrm{BaF}_{2}\right)$ either were too slow or had limited stopping power (sensitivity), the overall performance of such systems was not superior to that of conventional PET scanners (50). Also, the stability of the system for maintaining a good timing resolution over long periods was limited. A prototype TOF PET system based on $\mathrm{BaF}_{2}$ scintillators was developed and achieved a time resolution of 540 ps. With more recent improvements in PET detector technology, electronics, computer performance, and image processing, over the last few decades it became feasible to measure the arrival time of a photon in the scintillator to within a few hundred picoseconds. Thus, TOF PET was revived as PMTs with a short transit time and a fast rise time became more widespread and scintillators such as LSO or lutetium yttrium oxyorthosilicate, that have a short scintillation decay time and a high light yield, became available and cost-effective.

Theoretically, an intrinsic detector time resolution of about 15 ps would render the PET image reconstruction algorithm obsolete by identifying the location of an annihilation event to within $2 \mathrm{~mm}$ (Fig. 9D). However, PET detectors and electronics providing such superb time resolution are not yet available. Current clinical TOF PET systems achieve time resolutions of about 500 ps. However, the measured TOF information can be used to reduce noise by localizing annihilation events to within several centimeters (32). This is especially important for whole-body imaging of larger patients, in whom the ratio between true and scattered counts is unfavorable.

Tests with LSO scintillators show that a coincidence timing resolution down to 300 ps can be achieved with 2 single LSO crystals in coincidence (51). The improvement gained in the signal-to-noise ratio $\left(\mathrm{SNR}_{\mathrm{TOF}}\right)$ with a PET system using TOF, compared with non-TOF PET, is described by the following (52):

$$
\mathrm{SNR}_{\mathrm{TOF}}=\sqrt{\frac{\mathrm{D}}{\mathrm{dX}}} \times \mathrm{SNR}_{\text {non-TOF. }} \quad \text { Eq. } 1
$$

In this context, a 300-ps coincidence time resolution would result in a localization uncertainty $\mathrm{dX}$ of $4.5 \mathrm{~cm}$ and thus improve the $\mathrm{SNR}_{\mathrm{TOF}}$ by a factor of 3 for a $40-\mathrm{cm}$ diameter 

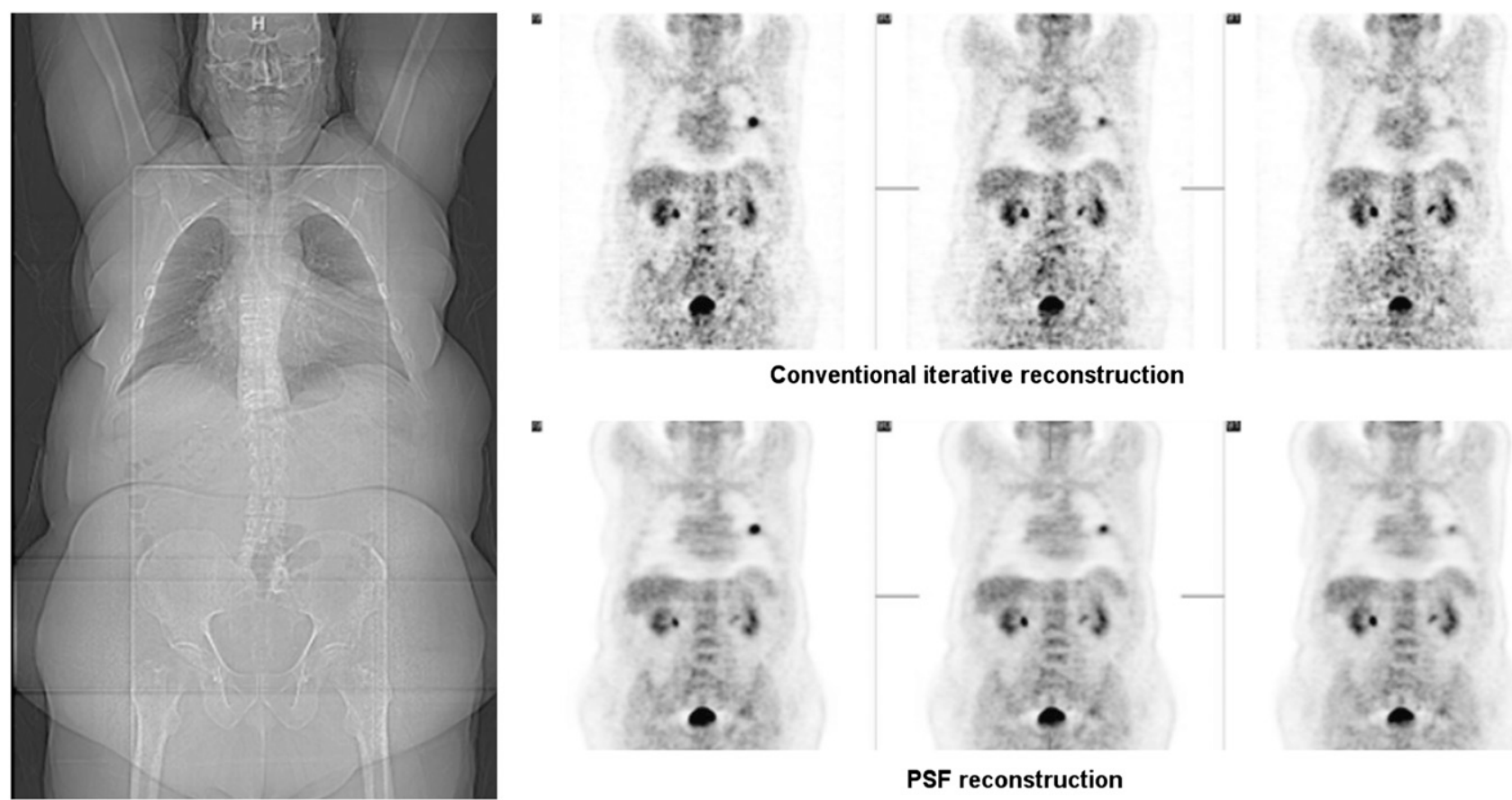

\section{Conventional iterative reconstruction}
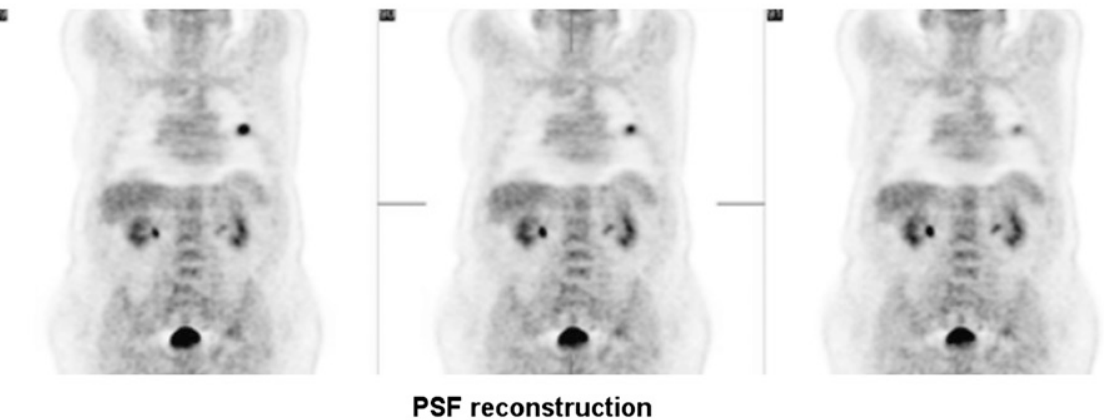

FIGURE 8. Advanced reconstruction algorithms attempt to include information about scanner geometry and properties for improving image quality. Point spread function, which is specific for a certain scanner, can be used to significantly improve image quality in terms of noise and contrast. Effect in obese patients is increased because ratio of true and random events is unfavorable. Example of patient with body mass index of $44.2(144 \mathrm{~kg})$ shows clear benefit of advanced reconstruction algorithm using point spread function. (Courtesy of Siemens Medical Solutions.)

$(D)$, compared with a non-TOF system ( $\left.\mathrm{SNR}_{\text {non-TOF}}\right)$. However, Equation 1 applies only to a uniform distribution of activity and not necessarily to a patient.

The potential of TOF PET lies in improving image quality in PET scans of obese patients, whereas the gain of SNR is modest for slim or normal-weight patients (Fig. 10). According to Equation 1, an improvement in SNR of 2-fold for 40-cm phantoms and 2.5-fold for $60-\mathrm{cm}$ phantoms can be achieved with this system.

Kuhn and Surti $(53,54)$ are developing a TOF system based on a fairly new scintillator material, $\mathrm{LaBr}_{3}$, which has a scintillation decay time of only $25 \mathrm{~ns}$, compared with the $40 \mathrm{~ns}$ of LSO, and is approximately twice as bright. However, this approach is still investigational, and its challenges include the hygroscopic characteristics of $\mathrm{LaBr}_{3}$, a stopping power less than that for LSO, and limited production capabilities (55).

\section{CT AND PET/CT}

$\mathrm{CT}$ is among the leading imaging modalities in clinical radiology. Although the low sensitivity of $\mathrm{CT}$ precludes its use beyond morphologic imaging, CT offers several advantages over other imaging modalities. First, it provides an

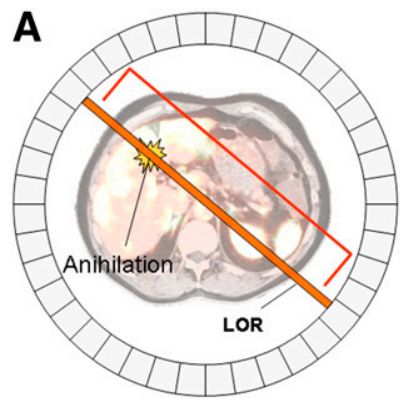

no TOF

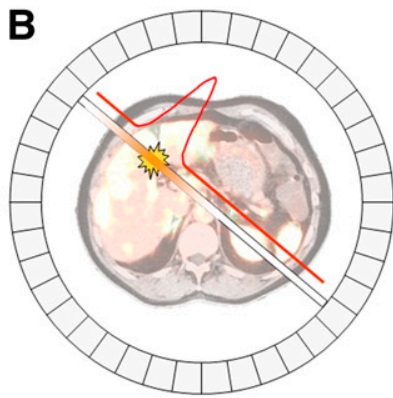

$\sim 500$ ps TOF resolution

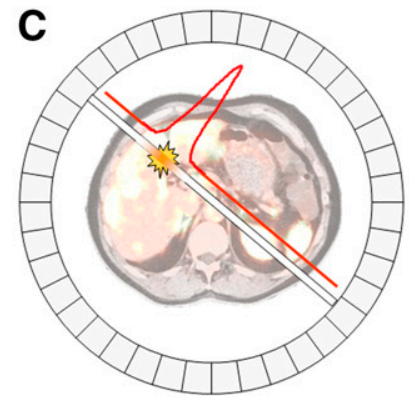

$\sim 300$ ps TOF resolution

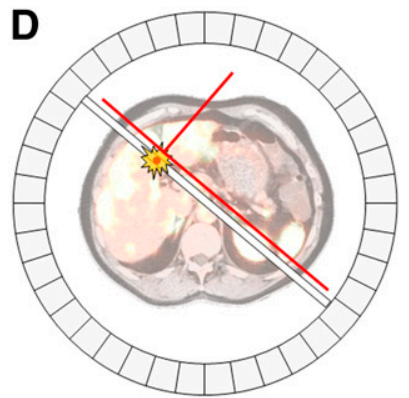

$\sim 15$ ps TOF resolution

FIGURE 9. Ability of PET scanner to accurately measure time between $2 \gamma$-interactions from 1 annihilation is defined as TOF capability. If no TOF information is available (time resolution $>1,500 \mathrm{ps}$ ), probability that coincidence occurred along 1 LOR is basically same (A). However, if time resolution can be increased ( $<600-800 \mathrm{ps}$ ), location of annihilation can be narrowed to several centimeters (B and $C$ ). In ideal PET system, where time resolution would be good (e.g., 15 ps), position of annihilation could be determined within several millimeters and would no longer require image reconstruction (D). (Courtesy of University of Tübingen.) 


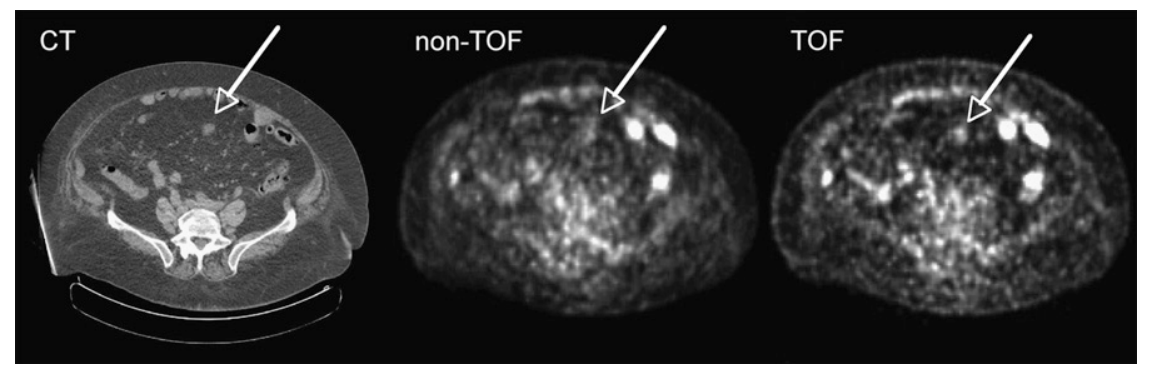

FIGURE 10. TOF PET images acquired with Phillips Gemini TF PET scanner. One can see that obese patient (119 kg; BMI, 46.5) particularly benefits from information gained because of time resolution of 600 ps. (Courtesy of Philips Medical Systems.)

excellent spatial anatomic resolution, in the millimeter and submillimeter range. Second, it is a fast modality allowing whole-body images to be acquired within a few seconds. These fast-acquisition protocols permit electrocardiographygated cardiac imaging and respiration-gated chest imaging. New CT technology has moved toward more detector rows, which is an advantage for gated imaging applications such as in cardiology. Currently, the state of the art is a 64-slice CT system that can obtain a whole-body scan within a single breath-hold and allows cardiac imaging (56-61), acquiring more than 150 slices per second at a resolution below 0.5 $\mathrm{mm}$. In clinical studies, devices acquiring up to 256 slices per rotation are currently under investigation (60). Another milestone is the introduction of dual-energy CT scanners. This new technology enhances image quality and improves differentiation of soft tissue and segmentation of tissue and iodine-based contrast agents. Better tissue contrast could especially improve the diagnostic value of CT in vascular diseases through early detection of calcifications $(62,63)$.

Although stand-alone CT does not play a significant role in molecular imaging, it complements PET by providing high-resolution anatomic information (24,25,64-67). Lowresolution CT scans are routinely used for PET attenuation correction. The combination of advanced CT scanner technology, such as dual-source CT, with a PET scanner might be beneficial for reducing the radiation dose (68) and improving the accuracy of PET attenuation correction, especially when iodine-based contrast agents are applied (69).

The latest commercial PET/CT technology combines up to 64-slice CT scanners with high-resolution 3D PET systems. The PET scanners often use TOF technology or image reconstruction based on the point spread function to achieve images with low noise, the highest spatial resolution, and good contrast. Fast scintillators, electronics with high counting-rate performance, 3D acquisition modes, and large axial fields of view of up to $22 \mathrm{~cm}$ can reduce PET scanning times to $1 \mathrm{~min}$ per bed position for slim patients and up to $4 \mathrm{~min}$ for obese patients.

\section{OPTICAL IMAGING}

Optical molecular imaging technologies use light emitted through fluorescence or bioluminescence (70). For fluorescence imaging, excitation light penetrates tissues and reaches a targeted reporter molecule in living subjects, resulting in the emission of light of lower-energy wavelengths, which is registered by a charge-coupled device (CCD) camera. In bioluminescence imaging, light is emitted through an enzymatic reaction, most frequently involving firefly luciferase, which oxidizes D-luciferin in the presence of adenosine triphosphate and oxygen. The result is an emission of yellow-green light at a wavelength of about $575 \mathrm{~nm}$. Cells expressing luciferase in vivo can be detected with a cooled, low-electronic-noise CCD camera after systemic injection of D-luciferin $(71,72)$.

In contrast to fluorescence, bioluminescence in vivo imaging has essentially no background signal. However, because of the limited penetration of light in tissue, the large amount of photon scattering, and the need to transfect cells, neither bioluminescence nor fluorescence imaging is likely to become widely applicable in cancer patients. Absorption of fluorescence light, which is predominant at wavelengths below $600 \mathrm{~nm}$ and above $800 \mathrm{~nm}$, is caused mainly by hemoglobin and water, 2 major constituents of living tissue. Thus, strategies are being developed to design specific imaging probes based on near-infrared fluorescence that detect lesions a few centimeters deep in tissue. Besides the optimization of biomarkers, efforts are currently focused on the improvement of optical imaging instrumentation by developing tomographic fluorescence imaging systems (73-75).

Nevertheless, some clinical applications of optical imaging are emerging. For instance, it has been used for detecting superficial malignant skin lesions. Another potential application is the detection of breast cancer, an idea that dates back more than 70 y (76) and was termed transillumination. Although tumor lesions were detectable, a clear differentiation between malignant and benign lesions was not always possible.

Important progress in in vivo optical imaging was made by introducing diffuse optical tomography, a technology based on diffuse light that penetrates tissue at multiple projections, yielding tomographic images. Diffuse optical tomography can provide quantitative information about light absorption, scatter, and uptake of fluorescence contrast agents (77). Currently, a major supplier of imaging technology is working on an advanced breast scanner based on fluorescence optical imaging (Fig. 11). This optical mammography system illuminates the breast with laser light 


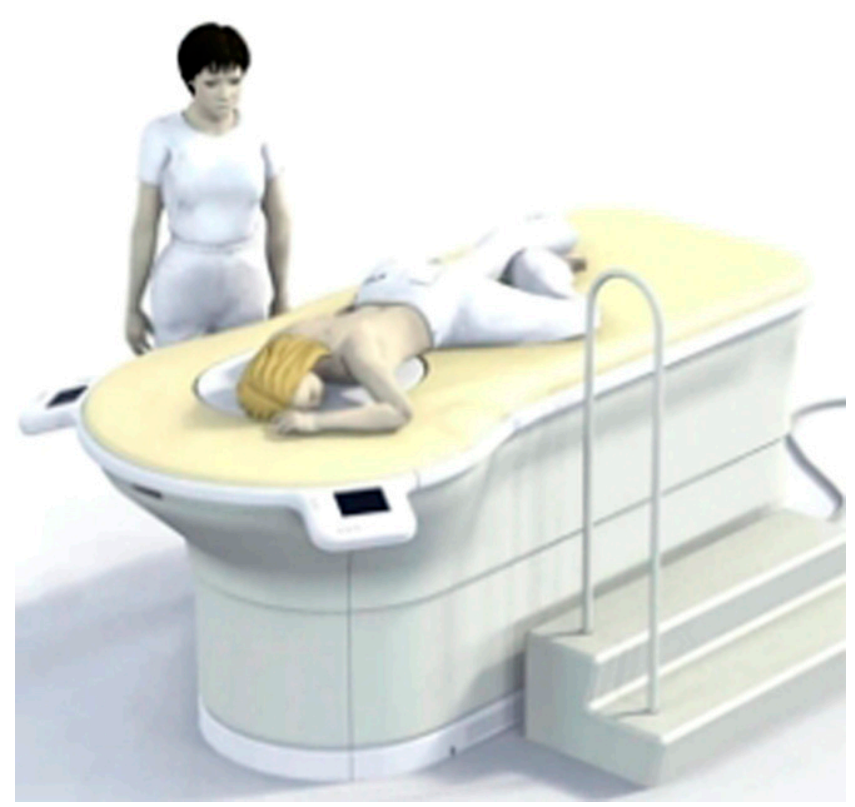

FIGURE 11. Optical mammography. Optical imaging, which is widely used in field of preclinical imaging, can successfully be applied for mammography applications in which high resolution is desirable but field of view is smaller. (Courtesy of Philips Medical Systems.)

from all sides and detects the fluorescence signal from the specific contrast agents applied. Dedicated reconstruction algorithms provide 3D images of the breast disease. This project is closely linked to a pharmaceutical company that develops specific fluorescence probes for breast imaging, indicating the importance of jointly developing imaging probes and the required technology.

\section{MRI}

\section{Fundamentals of MRI}

MRI is well integrated in the clinical practice of radiology and oncology (Fig. 12). MRI offers superior soft-tissue contrast and resolution in the submillimeter range, but its sensitivity for detecting imaging probes is roughly $10^{6}$ times lower than that of PET. This lower sensitivity often leads to the necessity of correlating functional information from PET with anatomic landmarks from MRI through image fusion. For image generation in MRI, the hydrogen nuclei, which are found in water, produce the detected signal. In some specialized MRI protocols, other nuclei (e.g., ${ }^{31} \mathrm{P},{ }^{13} \mathrm{C}$, and ${ }^{3} \mathrm{He}$ ) are also used for signal formation. Unlike PET and CT, MRI does not involve ionizing radiation. In fact, no known health hazards are associated with magnetic field exposure, provided there are no ferromagnetic objects present, such as metal implants or pacemakers (78). The high soft-tissue contrast of MRI is due to the relaxation properties of the coupled nuclei in the respective tissue and the presence of contrast medium. These differences were previously exploited in 1971 by Damadian, who found that relaxation times in cancerous tissue samples
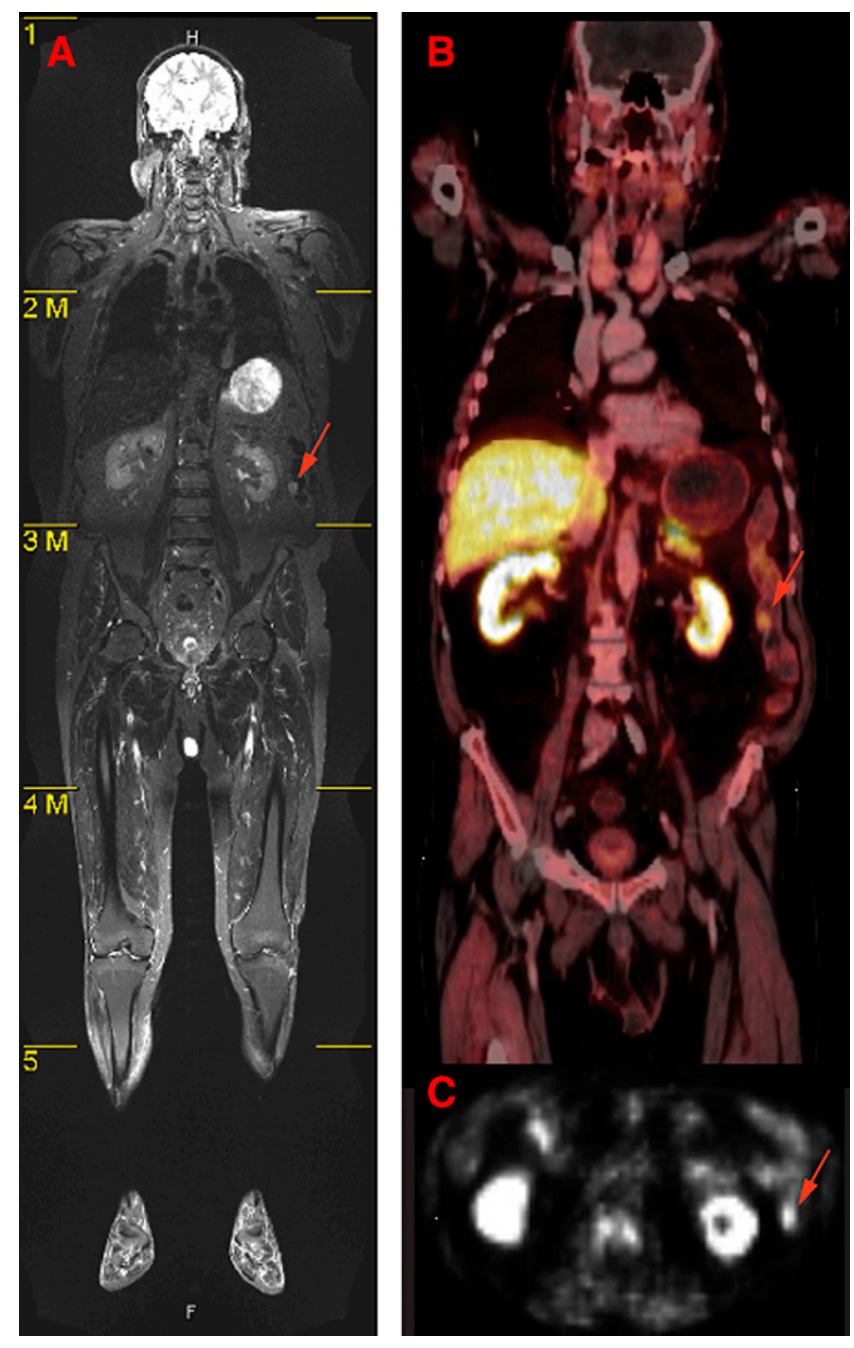

FIGURE 12. (A) High soft-tissue contrast of MR image is apparent in whole-body MRI tomogram of human. (B) In contrast, fused PET/CT image shows mainly bone structures. Metabolic PET images (B and C) clearly depict tumor area (arrow) that appears also in MR image. Anatomic information is most prominent in MR image, whereas PET image can help to guide diagnostic focus toward abnormalities in metabolism. These abnormalities can then also be identified as structural malignancies in PET images. (Courtesy of Heinz-Peter Schlemmer, University of Tübingen.)

from rats were longer than those in noncancerous tissue (79). MRI also allows arbitrary orientation of the imaged planes with respect to the standard anatomic orientations, enabling the imaging to focus on certain areas of interest for diagnostic purposes. Additionally, 3D scanning techniques using nearly isotropic voxels are routinely applied. Such datasets can then be resliced and used for various tasks such as tumor volumetrics, presurgical or intervention planning, radiation therapy planning, or therapy response evaluation $(80)$.

The enormous potential of MRI is based mainly on the variety of different contrasts that can be achieved by altering the scanning parameters, such as the echo and 
repetition times or the flip angle of the applied sequence. The generated images are usually either T1- or T2weighted or have some form of mixed contrast (Fig. 12). In tumors, prolonged $\mathrm{T} 1$ and $\mathrm{T} 2$ relaxation times can be observed; these are usually attributed to a large accumulation of extracellular water as a consequence of disordered cell growth and micronecrosis. These increased T2 values tend to appear hyperintense in T2-weighted images (81). Therefore, in some cases, tumors can be identified on unenhanced images. Specifically in the field of brain imaging, MRI contrast agents are routinely applied. Most of these contrast agents use complexes of the paramagnetic element gadolinium. Gadolinium cannot be directly visualized in MR images, but it shortens T1 (and to some extent T2) relaxation times (82). When the blood-brain barrier is disrupted - such as by intracranial mass lesions-the contrast agent penetrates the damaged barrier and enters the extracellular space, giving the appearance of increased intensity on T1-weighted images. Besides providing simple enhancement of certain pathologic structures, contrast agents also offer the opportunity for dynamic studies. Using fast MRI, the time course of the contrast agent signal can be tracked, allowing for measurements of mean transit times, regional cerebral blood volume, and regional cerebral blood flow (83-85).

\section{Beyond Anatomic MRI-Toward Functional Imaging}

The potential of MRI goes beyond anatomic imaging: for instance, MR spectroscopy can be used to dissect the molecular composition of tissues (Fig. 13) by applying selective radiofrequency excitation pulses such as stimulated echo acquisition mode ( 86 ) or point-resolved spectroscopy $(87,88)$. The Fourier transformation of the acquired signal provides a defined spectrum that allows for discrimination between various metabolites. Several studies have reported a reduction in the signal intensity of creatine and $N$ acetylaspartate and an increased level of the choline signal in brain tumors (89-94). The amount of tumor infiltration is proportional to the decrease in $\mathrm{N}$-acetylaspartate and the increase in choline signal $(95,96)$. Moreover, spectroscopic images of entire regions can be generated for preoperative staging of gliomas (97), $\mathrm{pH}$ imaging (98), monitoring of temperature (99), or evaluation of lactate changes during brain activation (100). Most of these studies are performed using ${ }^{1} \mathrm{H}$ spectroscopy. However, other nuclei could also be used to study function; for example, ${ }^{31} \mathrm{P} \mathrm{MR}$ spectroscopy can be used to study the energy status of the myocardium (101).

Because of their low abundance in humans, nonhydrogen nuclei exhibit a low SNR in MRI. However, signals can be enhanced by a technique called hyperpolarization, which involves polarizing certain nuclei, thus increasing their observed MRI signal by several orders of magnitude. For instance, ${ }^{3} \mathrm{He}$ and ${ }^{129} \mathrm{Xe}$ are polarized via optical pumping whereas ${ }^{13} \mathrm{C}$ can be polarized via parahydrogen-induced polarization $(102,103)$ and dynamic nuclear polarization (104,105). Applications of ${ }^{3} \mathrm{He}$ and ${ }^{127} \mathrm{Xe}$ include lung perfusion studies $(106,107)$. For ${ }^{127} \mathrm{Xe}$, there are also developments toward its use as a biomarker. Here, the high specificity of biochemical interactions is combined with the high sensitivity gained from hyperpolarization (108). ${ }^{13} \mathrm{C}$ has previously been used for MR angiography and perfusion studies in animals $(109,110)$. New endogenous contrast agents can be formed from hyperpolarized ${ }^{13} \mathrm{C}$ (e.g., ${ }^{13} \mathrm{C}$ [urea]); here, the administered substance does not change the $\mathrm{T} 1$ or $\mathrm{T} 2$ relaxation time, but similar to the case with a radioactive

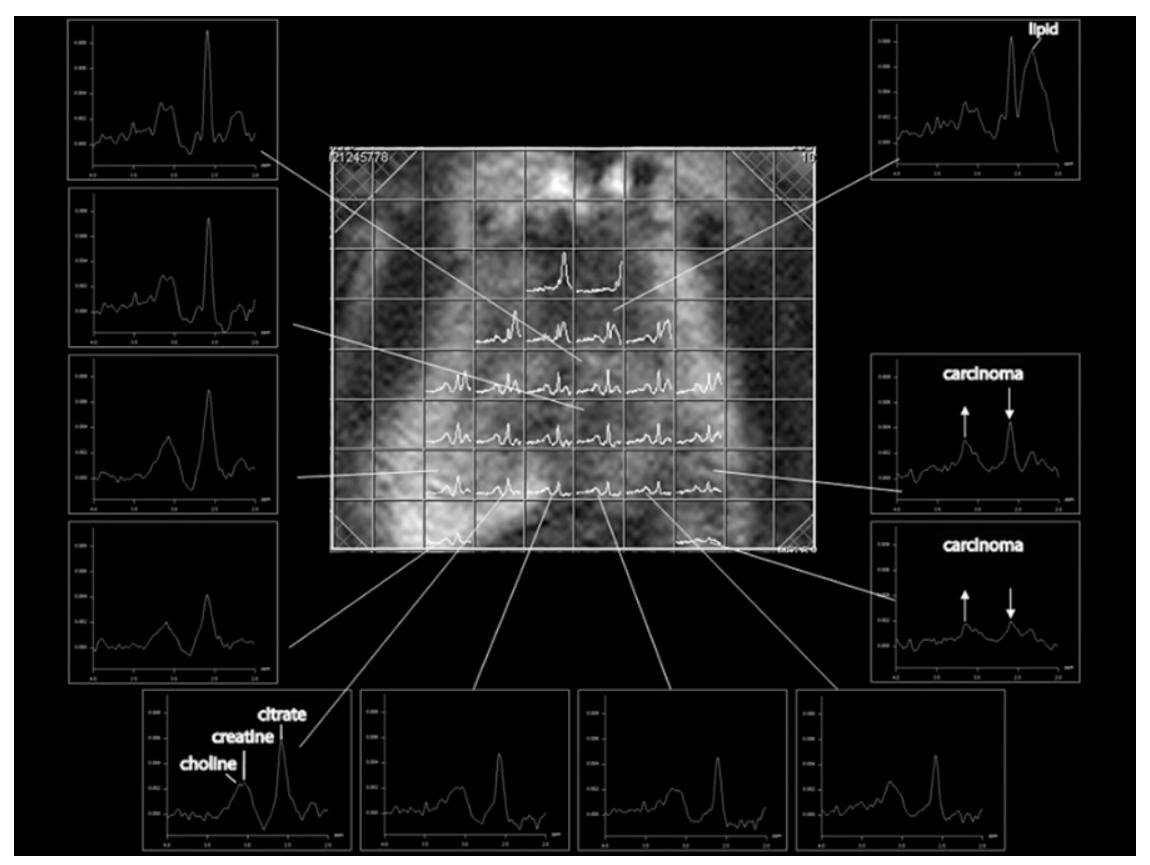

FIGURE 13. MR spectroscopy of prostate. Spectra are obtained from different voxels. Carcinogenic tissue can be differentiated by increase of choline signal and decrease of citrate signal. (Courtesy of Heinz-Peter Schlemmer, University of Tübingen.) 
tracer, the hyperpolarized nuclei are the source of the MRI signal. Consequently, hyperpolarized ${ }^{13} \mathrm{C}$ can be used for metabolic mapping. In a recent study, Golman et al. injected ${ }^{13} \mathrm{C}$-pyruvate in rats with an implanted P22 tumor and used MRI to noninvasively map the metabolic conversion of the injected pyruvate into alanine and lactate (Fig. 14). The approach has some similarities to ${ }^{18} \mathrm{~F}-\mathrm{FDG}$ PET studies, but despite the abundant presence of oxygen, these studies yielded the extra information that the cancer cells preferred anaerobic glycolysis (111). Through application of chemical shift imaging techniques with metabolic ${ }^{13} \mathrm{C}$ imaging, it seems feasible to distinguish signals from ${ }^{13} \mathrm{C}$ nuclei in different molecules - a possible advantage over PET and SPECT in some applications. A downside of hyperpolarized imaging is the relatively high costs and the rapid decay of the hyperpolarization; therefore, imaging of the injected ${ }^{13} \mathrm{C}$ substances must take place a few minutes after administration (112).

Functional processes in living subjects can also be studied via diffusion MRI. Here, a spatially varying magnetic field, generated by different gradients, is used to map phase differences in the MRI signal that are caused by diffusing molecules. Many MRI techniques can be made sensitive to diffusion by using adequate gradient pulses (113). Diffusion MRI has various potential clinical applications ranging from diagnosing ischemia (114), cancer $(115,116)$, multiple sclerosis (117), or Alzheimer's disease (118) to general fiber tracking via diffusion tensor imaging (119). Diffusion imaging is not restricted to the brain; it has also been applied in other regions of the body (e.g., for oncologic diagnosis), where it provides qualitative and quantitative insight about the tumor microenvironment and the integrity of cell membranes (120). Also, the use of functional diffusion imaging for the detection of neuro- nal activation that may be linked to cell swelling and membrane expansion has been explored $(121,122)$.

fMRI studies are frequently based on the BOLD contrast. This technique is based on the fact that the magnetic properties of oxygenated and deoxygenated hemoglobin in the blood are different and, therefore, produce different signals when imaged with $\mathrm{T} 2 *$-sensitive MRI sequences. The BOLD effect was first described by Ogawa in rat brain studies (123-125) and was subsequently described in human studies (126). Unlike contrast-enhanced MRI, BOLD contrast is a noninvasive technique because it uses endogenous information. A typical fMRI experiment for brain mapping uses either a block design, in which experimental conditions are separated into distinct blocks representing stimulation and baseline scenarios, or an event-related design, in which discrete, short events whose timing and order may be randomized are presented $(127,128)$. In both experimental types, the time course of the fMRI signal is recorded, usually with a T2*-sensitive echoplanar imaging sequence. Finally, the activation pattern is statistically extracted from the signal time course (129). BOLD fMRI is widely used for the study of different functional processes in the brain, including connections among brain regions, attention, memory, consciousness, vision, motor function, and others (130). The BOLD effect also has certain applications in cancer imaging to study tumor angiogenesis (131) or tumor oxygenation (132). In addition, it has been used for the presurgical planning of tumor resections (133).

\section{Toward Higher Field Strengths}

Today, most clinical MRI examinations are performed in field strengths on the order of $1.5 \mathrm{~T}$; however, in recent years, higher magnetic flux densities from 3 to $9.4 \mathrm{~T}$ have

FIGURE 14. Example of hyperpolarized ${ }^{13} \mathrm{C}$ imaging. Transverse proton reference image before and after introduction of gadolinium contrast agent in rat bearing 2 R3230 mammary adenocarcinomas (red arrows). NMR signal obtained simultaneously from alanine, lactate, and pyruvate is projected on anatomic ${ }^{1} \mathrm{H}$ images. High alanine concentration is seen in skeletal muscle adjacent to spinal cord, and the 2 tumors are indicated by high lactate and pyruvate signals. In tumor, lactate is twice as intense as pyruvate signal; in muscle, ratio of lactate to pyruvate is 1:1. (Courtesy of Jan Henrik Ardenkjaer-Larsen, GE Healthcare.)

Proton reference Image

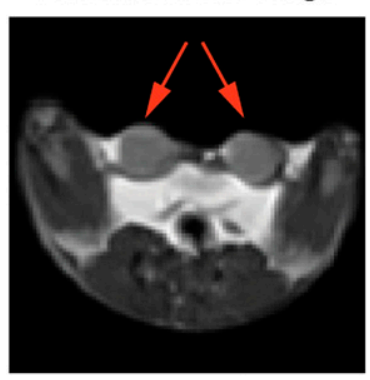

Pre Gd contrast agent

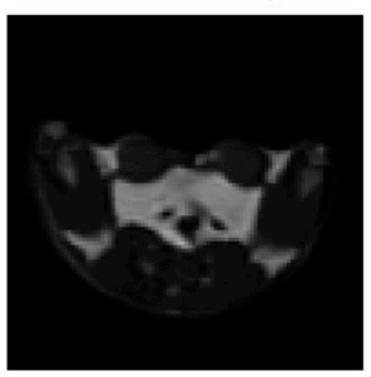

Alanine

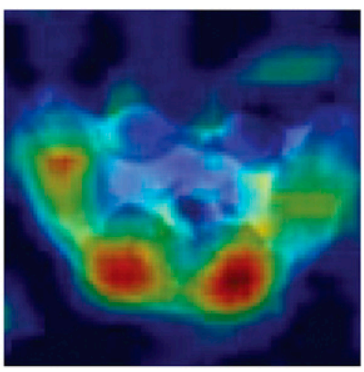

Post Gd contrast agent

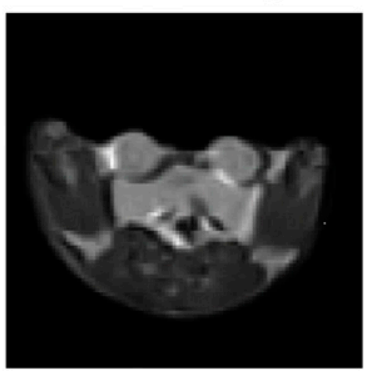

Lactate

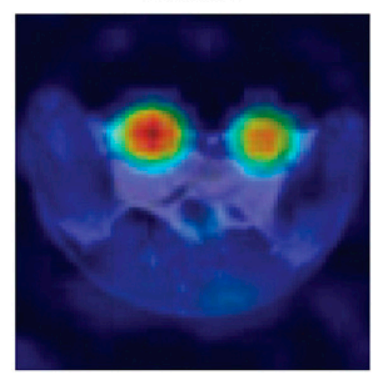

Pyruvate

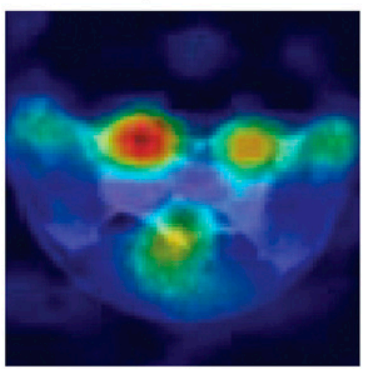


been introduced. High-field MRI has the potential to improve image quality and resolution and to reduce imaging time because the SNR increases proportionally with field strength. However, several problems arise from these higher magnetic field strengths (134), initiating the first discussions about the usefulness, even for human brain imaging, of magnets exceeding $7 \mathrm{~T}$. A major problem is that the applied radiofrequency signal is also proportional to the main magnetic field strength $\left(B_{0}\right)$; therefore, the radiofrequency wavelength for a higher $B_{0}$ is reduced to dimensions that are similar to the human body. These dielectric effects lead to interferences of superimposed radiofrequency waves inside the body, which can result in an almost complete cancellation of the radiofrequency excitation in parts of the pelvis or abdomen already at field strengths of $3 \mathrm{~T}$. Additionally, the amount of radiofrequency energy deposited in the body is a concern at high field strengths. Nevertheless, spectroscopy, chemical-shift imaging, and susceptibility imaging clearly benefit from the higher $\mathrm{B}_{0}$ at $3 \mathrm{~T}$, compared with $1.5 \mathrm{~T}$ (135). Further improvement, especially of spectroscopic and fMRI, is seen at field strengths of between 7 and $9.4 \mathrm{~T}$, especially when dedicated radiofrequency coils are used $(136,137)$. At $7 \mathrm{~T}$, however, signal-to-noise inhomogeneities inside the head are present (138). New techniques such as parallel transmissions that include a homogeneity correction for the radiofrequency field ( $\mathrm{B}_{1}$ shimming) are used to correct for radiofrequency nonuniformities (139). These approaches may also solve some problems that arise from the higher radiofrequency power deposition at high- $\mathrm{B}_{0}$ fields. Many problems in high-field MRI might be solved by the use of techniques to receive parallel MRI signals and parallel transmissions. These techniques use the information provided by several coil elements to accelerate image acquisition and apply them during signal transmission to compensate for nonuniformities in the radiofrequency field $(140,141)$. The dielectric effects are not as pronounced in small-animal MRI because of the smaller object size. Therefore, dedicated animal MRI systems that are suitable for mice and rats operate at $\mathrm{B}_{0}$ field strengths of between 4.7 and $16.4 \mathrm{~T}$. Further improvements in image quality are to be expected by the implementation of parallel-reception and -transmission techniques, as well as cryo-coils, which are cooled with liquid helium or nitrogen and yield signal-to-noise improvements of approximately a factor of 2 , compared with coils operated at room temperature (142).

\section{Whole-Body MRI}

Though MRI is a diverse diagnostic modality, wholebody scans of patients were not feasible in the past because repeated examinations with patient repositioning and separate coils were needed to cover all parts of the body. The advent of parallel imaging techniques, such as generalized autocalibrating, partially parallel acquisitions (143) and sensitivity encoding (144) has accelerated image acquisition times, thus allowing whole-body MRI examinations with high spatial resolution (145). Initial results show that whole-body MRI is a promising modality in oncology, especially for the detection of metastases and hematologic malignancies (146). Performing whole-body MRI similarly to a CT examination, with continuous table motion, is likely to improve time efficiency (147).

\section{MULTIMODALITY IMAGING: PET/MRI}

\section{We Have PET/CT_Do We Need PET/MRI?}

In the late 1990s, hybrid PET/CT was introduced, a concept that was so successful $(24,25,64,65,67,148)$ that stand-alone PET systems are almost no longer commercially available. The main shortcomings of PET/CT are based on the way this combination is realized and on the intrinsic characteristics of CT. Although the 2 modalities are hard-wired using a common patient bed in PET/CT scanners, PET/CT does not allow simultaneous acquisition of CT and PET data. Instead, the patient is first scanned by CT and then by PET. Therefore, some misalignment between CT and PET images due to patient or organ motion cannot be ruled out, potentially leading to artifacts in the fused images (149-151). In particular, the fact that CT scans are acquired quickly and PET images are acquired during an extended period of the respiratory cycle can lead to artifacts $(24,152)$. The lack of simultaneous imaging also prevents the temporal merging of information acquired from PET and CT. Moreover, radiation doses are an inherent problem of PET/CT, although the risk for patients, if appropriately selected for imaging, is likely low (153). Finally, the soft-tissue contrast of CT images is limited, especially when no intravenous contrast agents are applied.

The development of hybrid PET/MRI systems started in the late 1990s (154). In contrast to CT, MRI offers a multitude of endogenous contrasts and a high capability of differentiating soft tissues, as well as many exogenous contrast media ranging from gadolinium-based agents to highly specified cellular markers. With a sensitivity in the picomolar range, PET is ideally suited for the visualization of specific molecules in living organisms. However, PET lacks the spatial resolution offered by MRI, which in turn lacks sensitivity. Therefore, the combination of PET and MRI is highly complementary. In contrast to CT, no additional radiation results from MRI. Furthermore, as mentioned previously, MRI has interesting functional imaging capabilities, such as perfusion, BOLD effect, diffusion, and spectroscopy. The combination of PET and MRI is feasible either in a PET/CT-like combination, in which images are obtained serially, or in a true integrated PET/MRI system that permits simultaneous scanning. The latter offers the advantage of reduced data acquisition times (because the MRI scan is performed during the PET scan) and enables dual functional imaging in which physiologic processes are studied isochronously. These advantages allow either crosscorrelation studies, in which the same physiologic parameter is evaluated in PET and MRI (e.g., perfusion), or experi- 
ments in which 2 functional parameters are simultaneously evaluated. For example, this technique could monitor perfusion or tracer kinetics using PET and diffusion, spectroscopy, or the BOLD effect using MRI.

\section{Technical Aspects}

Traditional PET systems use PMTs to detect the scintillation light. However, PMTs are sensitive to magnetic fields and are therefore not functional inside an MRI scanner To overcome this problem, various approaches to the combination of PET and MRI have been established. Optical fibers can be used to lead the light from the scintillation crystals outside the fringe field of the magnet to the PMTs (154-156). Split magnets, with the PET detector between the 2 magnet halves using light fibers (157), or field cycling systems, which switch off the field of the magnet during PET acquisition $(158,159)$, are being built. Long optical fibers, however, result in a loss of light and consequently lower performance of such a PET system. This loss can be overcome by the use of magnetic-field-compatible solidstate light detectors, such as APDs $(41,45)$. This approach also permits easier expansion of the axial field of view of the PET system (42). In addition, an approach using both short optical fibers and solid-state light detectors has been built (43). The above-mentioned systems were first implemented in small-animal imagers, and in vivo pilot studies using these systems have been reported by various groups $(42,43,155,156)$. Recently, an APD-based system for simultaneous PET/MRI human brain imaging was built and used to acquire the first simultaneous human PET/MRI scans $(160,161)$ (Fig. 15). The mutual interference between PET and MRI is a critical problem; MRI can affect PET performance because of the high magnetic field, gradient fields, and radiofrequency field. MR image quality, however, can be impaired by either radiofrequency noise introduced by the PET electronics or magnetic field inhomogeneities caused by the presence of different materials in the PET insert and eddy currents induced from the gradient

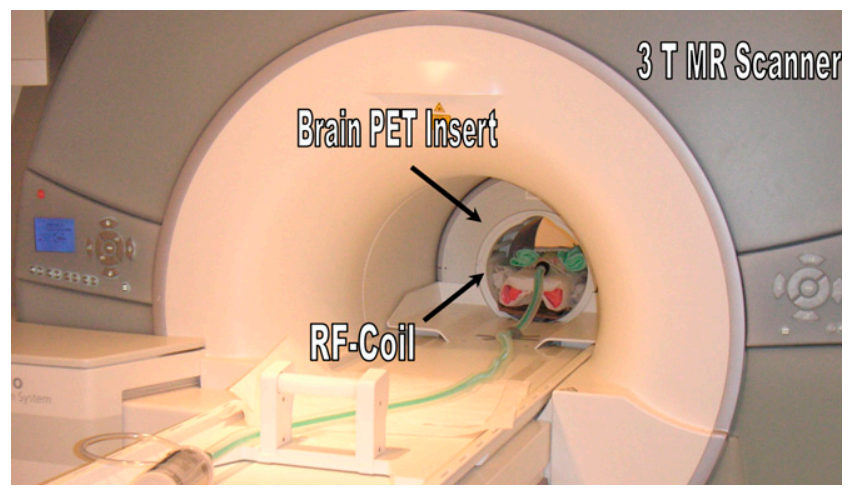

FIGURE 15. First installation of clinical PET/MRI system at University of Tübingen. System is dedicated for brain applications and features 3-T MRI system with dedicated head coil and PET head insert with $18-\mathrm{cm}$ axial field of view. (Courtesy of University of Tübingen.) system in the conducting structures of the PET housing and circuit boards. Moreover, the operating temperature needs to be stabilized to ensure reliable PET and MRI performance. Overcoming these technical hurdles is a challenging but worthwhile goal. Figure 16 shows the small-animal PET/MRI system developed by our group $(42,45)$. The 10 PET detectors are integrated in a 7-T small-animal MRI scanner. Inside the PET ring, a quadrature MRI coil is installed for MRI signal transmission and reception. The combined PET/MRI field of view is $35 \mathrm{~mm}$ in the transaxial direction and $19 \mathrm{~mm}$ in the axial direction, allowing for simultaneous PET/MRI studies in mice. Our initial results show that neither PET nor MRI performance is fundamentally impaired by this approach (42).

Performing PET attenuation correction with MR image information is another challenge (162). Proposed solutions have included sophisticated atlases and algorithms or are

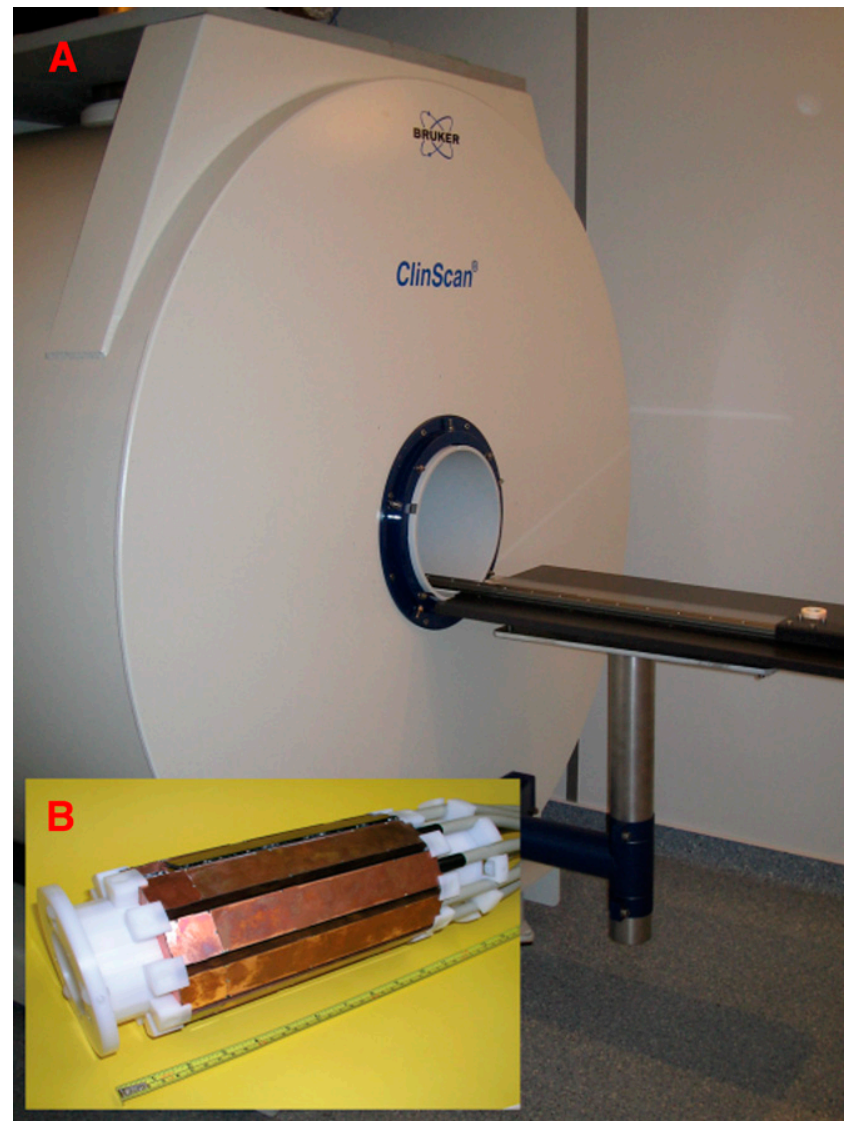

FIGURE 16. (A) Combined PET/MRI is performed using 7-T small-animal MRI scanner with gradient strength of $300 \mathrm{mT} / \mathrm{m}$. (B) PET insert consisting of 10 detector modules, developed and constructed by our group, is installed inside bore of magnet. Inside PET insert is placed $35-\mathrm{mm}$ quadrature coil for MRI transmission and reception. Combined PET/MRI field of view is approximately $35 \mathrm{~mm}$ in transaxial direction and $19 \mathrm{~mm}$ in axial direction. Simultaneous PET/MRI studies, which allow combination of function with anatomy or of PET functional imaging with MRI functional imaging, can be performed using this system. (Courtesy of University of Tübingen.) 
CT

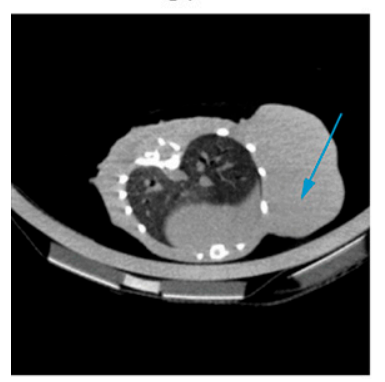

MR

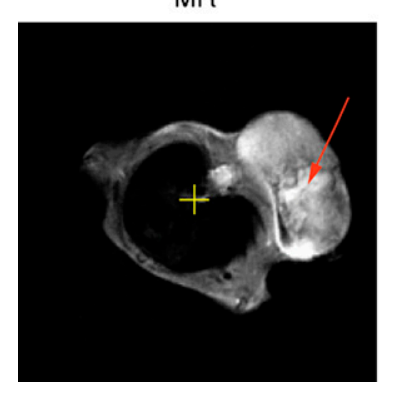

PET

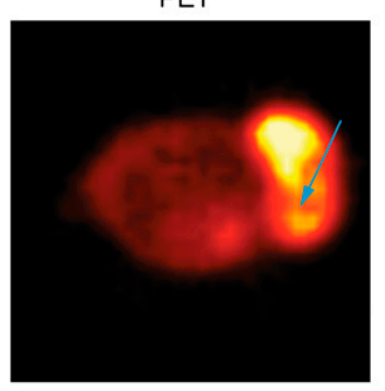

PET

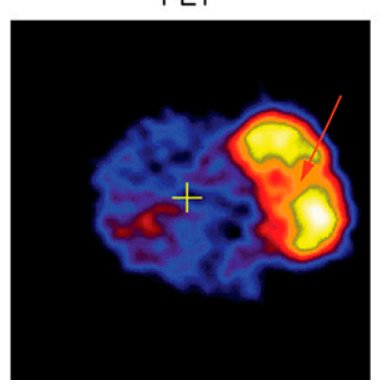

$\mathrm{PET} / \mathrm{CT}$

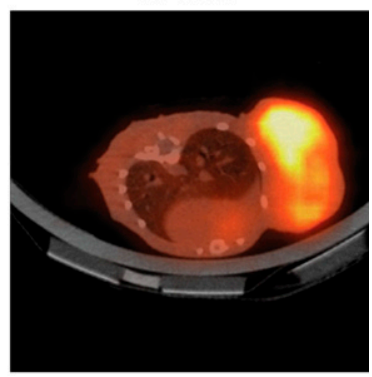

$\mathrm{PET} / \mathrm{MR}$

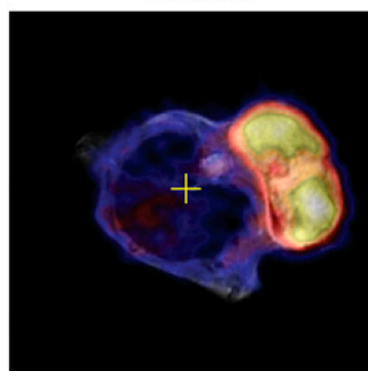

FIGURE 17. Images of 2 BALB/c mice bearing CT26 tumor. In combination PET/ CT (first row), region of low tracer uptake in tumor (blue arrow) cannot be explained by CT images, because entire tumor appears as homogeneous tissue. In sharp contrast, T2-weighted MR images obtained by simultaneous PET/MRI reveal hyperintense area corresponding to area of low tracer uptake (red arrow). This is indication of tumor necrosis. From these images, one domain in which CT excels is also visible: inside lung, MR image depicts nearly no structures because of lack of signal-generating protons; in contrast, CT images reveal details of lung bronchia. (Courtesy of University of Tübingen.) based on machine learning approaches (163). Also, special MRI sequences using ultrashort echo times to depict bone structures seem feasible as a basis for PET attenuation correction. It currently remains unclear which approach will be the most useful, and it is more than probable that a combination of different methods will be adopted.

Because the PET insert is operated inside the magnet, the spatial resolution of PET images may be improved because of the reduction of the positron range, as is especially important for small-animal systems. Particularly for highenergy positron emitters (e.g., ${ }^{15} \mathrm{O}$ ), the path the positron travels before annihilation may be reduced by the spiral movement induced by the magnetic field (164).

Motion correction of PET images based on MR images is also a viable option. Here, special MRI sequences that detect the motion of the subject can be applied by either 1dimensional navigator scans or more elaborate techniques in 2 and 3 dimensions. Ideally, these protocols can be combined with the MRI sequence already running to provide motion information about the subject in intervals of as short as $1 \mathrm{~s}$. The motion vectors can than be extracted from the motion-sensitive MRI scans and made available to the PET system, allowing either online or postacquisition motion correction of the PET images.

\section{Applications: from Combining Function with Anatomy to Multifunctional Imaging}

The original idea behind merging PET and MRI was to combine the functional information provided by PET with the high soft-tissue contrast offered by MRI. Figure 17 shows a BALB/c mouse bearing a CT26 tumor and high-resolution PET images of tumor cell proliferation that have been obtained by injection of an ${ }^{18} \mathrm{~F}-3$-deoxy-3' fluorothymidine tracer. The morphology of the tumor areas with low tracer uptake remained unclear after evaluation of the PET images alone. Here, the MR images helped to identify necrotic regions. In contrast, only limited information about the tumor could be obtained using the CT scan of a similar mouse. Moreover brain studies benefit greatly from the additional morphologic information provided by MRI. Especially for PET quantification, a better determination of the region of interest (e.g., in the striatum) is possible from the anatomic high soft-tissue contrast of MRI. Furthermore, it seems possible to correct for partial-volume effects using accurate morphologic information $(165,166)$.

Because it is possible, as our own studies have shown, to operate a PET system simultaneously with an MRI scanner without sacrificing the performance of either modality, the prospects for PET/MRI extend far beyond just combining function with anatomy. In the field of cardiology, the huge potential of MRI to assess cardiac function can be combined with the possibilities offered by cardiac PET; this combination would allow doctors to assess the metabolic viability of the heart muscle and its perfusion. Initial studies combining MRI spectroscopy with PET have already been performed on isolated perfused rat hearts (167), but more elaborate cardiac PET/MRI studies involving cardiac stress simultaneously assessed with PET and MRI seem feasible. Dual functional studies correlating the same parameters (e.g., perfusion in PET via radioactive water or ammonia and in MRI using spin labeling or MRI contrast agents) can help to cross correlate and validate different acquisition techniques. Using the strengths of each individual modality, one can simultaneously assess different molecular parameters. For instance, diffusion processes may be tracked simultaneously with PET tracer uptake, or PET perfusion can be correlated with the MRI BOLD effect. Because of the large number of existing PET probes 
and the various functional imaging capabilities of MRI, the number of possible combinations for molecular imaging readouts is virtually unlimited. The advantage of truly simultaneous PET/MRI is that the same subject is scanned at the same time with identical environmental parameters and stimuli. It is likely that such functional studies will further push the limits for basic biologic research and will open new realms for studying biology in vivo.

\section{CONCLUSION}

Molecular imaging is an emerging field that is transforming clinical in vivo diagnostics from the anatomic to the functional domain.

The latest developments in PET detector technology and PET reconstruction algorithms have resulted in highresolution images that reveal small tumors and allow accurate quantification of biologic processes. Though optical imaging has great potential to be developed as a functional imaging modality, it might not reach the usefulness of PET or MRI in whole-body imaging because of restricted penetration and limited quantification accuracy.

Preclinical and clinical MRI applications have expanded beyond simple anatomic imaging into the domain of molecular imaging. New contrast agents and hyperpolarization techniques enhance the sensitivity of the MRI experiments, paving the way for more clinically oriented molecular imaging.

The trend has clearly gone toward functional and multimodality imaging for detecting diseases, because earlier diagnosis positively affects disease progression. A milestone in PET was the introduction of combined PET/CT. The first studies proved that PET/MRI has enormous potential as an integrated molecular imaging tool. Because of its high cost, it is unlikely to replace PET/CT in the clinical environment. However, molecular imaging research will benefit highly from the new opportunities offered by this combination. In general, the combination of different modalities such as PET, SPECT, optical imaging, ultrasound, magnetoencephalography, and CT with MRI may lead to new insights in research and novel clinical applications.

\section{REFERENCES}

1. Muehllehner G, Karp JS. Positron emission tomography. Phys Med Biol. 2006;51:R117-137.

2. Herbert DJ, Saveliev V, Belcari N, D'Ascenzo N, Del Guerra A, Golovin A. First results of scintillator readout with silicon photomultiplier. IEEE Trans Nucl Sci. 2006;53:389-394.

3. Holl I, Lorenz E, Natkaniez S, Renker D, Schmelz C, Schwartz B. Some studies of avalanche photodiode readout of fast scintillators. IEEE Trans Nucl Sci. 1995;42:351-356.

4. Pichler BJ, Böning G, Rafecas M, Schlosshauer M, Lorenz E, Ziegler SI. LGSO scintillation crystals coupled to new large area APDs compared to LSO and BGO. IEEE Trans Nucl Sci. 1999;46:289-291.

5. Schmelz C, Bradbury SM, Holl I, Lorenz E, Renker D, Ziegler S. Feasibility study of an avalanche photodiode readout for a high resolution PET with nsec time resolution. IEEE Trans Nucl Sci. 1995;42:1080-1084.

6. Nutt R. 1999 ICP Distinguished Scientist Award: the history of positron emission tomography. Mol Imaging Biol. 2002;4:11-26.
7. Chilton M. Planning and financing a PET center. J Nucl Med. 1991;32:35N, $38 \mathrm{~N}-41 \mathrm{~N}, 51 \mathrm{~N}$.

8. Phelps ME. Nuclear medicine, molecular imaging, and molecular medicine. J Nucl Med. 2002;43:13N-14N.

9. Ter-Pogossian MM, Phelps ME, Hoffman EJ, Mullani NA. A positron-emission transaxial tomograph for nuclear imaging (PETT). Radiology. 1975;114:89-98.

10. Karp JS, Surti S, Daube-Witherspoon ME, et al. Performance of a brain PET camera based on anger-logic gadolinium oxyorthosilicate detectors. $\mathrm{J} \mathrm{Nucl}$ Med. 2003;44:1340-1349.

11. Wienhard K, Dahlbom M, Eriksson L, et al. The ECAT EXACT HR: performance of a new high resolution positron scanner. J Comput Assist Tomogr. 1994;18:110-118.

12. Casey ME, Nutt R. A multicrystal two dimensional BGO detector system for positron emission tomography. IEEE Trans Nucl Sci. 1986;33:460-463.

13. Dahlbom M, Hoffman EJ. An evaluation of a two-dimensional array detector for high resolution PET. IEEE Trans Med Imaging. 1988;7:264-272.

14. Townsend DW, Geissbuhler A, Defrise M, et al. Fully three-dimensional reconstruction for a PET camera with retractable septa. IEEE Trans Med Imaging. 1991;10:505-512.

15. Cherry SR, Dahlbom M, Hoffman EJ. 3D PET using a conventional multislice tomograph without septa. J Comput Assist Tomogr. 1991;15:655-668.

16. Spinks TJ, Jones T, Bailey DL, et al. Physical performance of a positron tomograph for brain imaging with retractable septa. Phys Med Biol. 1992;37: 1637-1655.

17. Lartizien C, Comtat C, Kinahan PE, Ferreira N, Bendriem B, Trebossen R. Optimization of injected dose based on noise equivalent count rates for 2- and 3-dimensional whole-body PET. J Nucl Med. 2002;43:1268-1278.

18. Lewitt RM, Matej S. Overview of methods for image reconstruction from projections in emission computed tomography [abstract]. Proc IEEE. 2003; 91:1588.

19. Wang CX, Snyder WE, Bilbro G, Santago P. Performance evaluation of filtered backprojection reconstruction and iterative reconstruction methods for PET images. Comput Biol Med. 1998;28:13-24.

20. Shepp LA, Vardi Y. Maximum likelihood reconstruction for emission tomography. IEEE Trans Med Imaging. 1982;1:113-122.

21. Rafecas M, Boning G, Pichler B, Lorenz E, Schwaiger M, Ziegler SI. A Monte Carlo study of high-resolution PET with granulated dual-layer detectors. IEEE Trans Nucl Sci. 2001;48:1490-1495.

22. Schmitt D, Karuta B, Carrier C, Lecomte R. Fast point spread function computation from aperture functions in high-resolution positron emission tomography. IEEE Trans Med Imaging. 1988;7:2.

23. Karuta B, Lecomte R. Effect of detector weighting functions on the point spread function of high-resolution PET tomographs: a simulation study. IEEE Trans Med Imaging. 1992;11:379-385.

24. Beyer T, Antoch G, Blodgett T, Freudenberg LF, Akhurst T, Mueller S. Dualmodality PET/CT imaging: the effect of respiratory motion on combined image quality in clinical oncology. Eur J Nucl Med Mol Imaging. 2003;30:588-596.

25. Townsend DW, Carney JP, Yap JT, Hall NC. PET/CT today and tomorrow. J Nucl Med. 2004;45(suppl 1):4S-14S

26. Casey ME, Eriksson L, Schmand M, et al. Investigation of LSO crystals for high spatial resolution positron emission tomography. IEEE Trans Nucl Sci. 1997;44:1109-1113.

27. Ziegler SI, Pichler BJ, Boening G, et al. A prototype high-resolution animal positron tomograph with avalanche photodiode arrays and LSO crystals. Eur J Nucl Med. 2001;28:136-143.

28. McElroy DP, Hoose M, Pimpl W, Spanoudaki V, Schuler T, Ziegler SI. A true singles list-mode data acquisition system for a small animal PET scanner with independent crystal readout. Phys Med Biol. 2005;50:3323-3335.

29. Aykac M, Bauer F, Williams CW, Loope M, Schmand M. Timing performance of Hi-Rez detector for time-of-flight (TOF) PET. IEEE Trans Nucl Sci. 2006;53:1084-1089.

30. Brambilla M, Secco C, Dominietto M, Matheoud R, Sacchetti G, Inglese E. Performance characteristics obtained for a new 3-dimensional lutetium oxyorthosilicate-based whole-body PET/CT scanner with the National Electrical Manufacturers Association NU 2-2001 standard. J Nucl Med. 2005;46:20832091.

31. Teras M, Tolvanen T, Johansson JJ, Williams JJ, Knuuti J. Performance of the new generation of whole-body PET/CT scanners: Discovery STE and Discovery VCT. Eur J Nucl Med Mol Imaging. 2007;34:1683-1692.

32. Surti S, Kuhn A, Werner ME, Perkins AE, Kolthammer J, Karp JS. Performance of Philips Gemini TF PET/CT scanner with special consideration for its time-of-flight imaging capabilities. J Nucl Med. 2007;48:471-480.

33. Stickel JR, Cherry SR. High-resolution PET detector design: modelling components of intrinsic spatial resolution. Phys Med Biol. 2005;50:179-195. 
34. Stickel JR, Qi J, Cherry SR. Fabrication and characterization of a $0.5-\mathrm{mm}$ lutetium oxyorthosilicate detector array for high-resolution PET applications. J Nucl Med. 2007;48:115-121.

35. Wang Y, Seidel J, Tsui BM, Vaquero JJ, Pomper MG. Performance evaluation of the GE Healthcare eXplore VISTA dual-ring small-animal PET scanner. J Nucl Med. 2006;47:1891-1900.

36. Bergeron M, Cadorette J, Beaudoin J-F, et al. Performance evaluation of the LabPET(TM); APD-based digital PET scanner [abstract]. In: Cadorette J, ed. 2007 IEEE Nuclear Science Symposium Conference Record. Piscataway, NJ: IEEE; 2007:4185.

37. de Jong HW, van Velden FH, Kloet RW, Buijs FL, Boellaard R, Lammertsma AA. Performance evaluation of the ECAT HRRT: an LSO-LYSO double layer high resolution, high sensitivity scanner. Phys Med Biol. 2007;52:1505-1526.

38. Dokhale PA, Silverman RW, Shah KS, et al. Performance measurements of a depth-encoding PET detector module based on position-sensitive avalanche photodiode read-out. Phys Med Biol. 2004;49:4293-4304.

39. Wienhard K, Schmand M, Casey ME, et al. The ECAT HRRT: performance and first clinical application of the new high resolution research tomograph. IEEE Trans Nucl Sci. 2002;49:104-110.

40. Lecomte R, Cadorette J, Jouan A, Heon M, Rouleau D, Gauthier G. High resolution positron emission tomography with a prototype camera based on solid state scintillation detectors. IEEE Trans Nucl Sci. 1990;37:805-811.

41. Pichler B, Lorenz E, Mirzoyan R, et al. Performance test of a LSO-APD PET module in a 9.4 tesla magnet. In: 1998 IEEE Nuclear Science Symposium and Medical Imaging Conference. Piscataway, NJ: IEEE; 1999:1237-1239.

42. Judenhofer MS, Wehrl HF, Newport DF, et al. Simultaneous PET-MRI: a new approach for functional and morphological imaging. Nat Med. 2008;14:459-465.

43. Catana C, Wu Y, Judenhofer MS, Qi J, Pichler BJ, Cherry SR. Simultaneous acquisition of multislice PET and MR images: initial results with a MRcompatible PET scanner. J Nucl Med. 2006;47:1968-1976.

44. Pichler BJ, Judenhofer MS, Catana C, et al. Performance test of an LSO-APD detector in a 7-T MRI scanner for simultaneous PET/MRI. J Nucl Med. 2006; 47:639-647.

45. Judenhofer MS, Catana C, Swann BK, et al. PET/MR images acquired with a compact MR-compatible PET detector in a 7-T magnet. Radiology. 2007;244: $807-814$

46. Otte AN, Barral J, Dolgoshein B, et al. A test of silicon photomultipliers as readout for PET. Nucl Instrum Methods Phys Res A. 2005;545:705-715.

47. Lange K, Carson R. EM reconstruction algorithms for emission and transmission tomography. J Comput Assist Tomogr. 1984;8:306-316.

48. Hebert TJ, Leahy R. Statistic-based MAP image-reconstruction from Poisson data using Gibbs priors [abstract]. IEEE Trans Acoust. 1992;40:2290.

49. Leahy R, Byrne C. Recent developments in iterative image reconstruction for PET and SPECT. IEEE Trans Med Imaging. 2000;19:257-260.

50. Lewellen TK. Time-of-flight PET. Semin Nucl Med. 1998;28:268-275.

51. Moses WW. Time of flight in PET revisited. IEEE Trans Nucl Sci. 2003; 50:1325-1330.

52. Cherry SR. The 2006 Henry N. Wagner Lecture: of mice and men (and positrons)—advances in PET imaging technology. J Nucl Med. 2006;47: 1735-1745.

53. Kuhn A, Surti S, Karp JS, et al. Design of a lanthanum bromide detector for time-of-flight PET. IEEE Trans Nucl Sci. 2004;51:2550-2557.

54. Surti S, Karp JS, Muehllehner G. Image quality assessment of LaBr3-based whole-body 3D PET scanners: a Monte Carlo evaluation. Phys Med Biol. 2004;49:4593-4610.

55. Nassalski A, Kapusta M, Batsch T, et al. Comparative study of scintillators for PET/CT detectors. IEEE Trans Nucl Sci. 2007;54:3-10.

56. Becker CR, Ohnesorge BM, Schoepf UJ, Reiser MF. Current development of cardiac imaging with multidetector-row CT. Eur J Radiol. 2000;36:97-103.

57. Nikolaou K, Flohr T, Knez A, et al. Advances in cardiac CT imaging: 64-slice scanner. Int J Cardiovasc Imaging. 2004;20:535-540.

58. Flohr TG, Ohnesorge BM. Imaging of the heart with computed tomography. Basic Res Cardiol. 2008;103:161-173.

59. Manghat NE, Morgan-Hughes GJ, Marshall AJ, Roobottom CA. Multi-detector row computed tomography: imaging the coronary arteries. Clin Radiol. 2005;60:939-952.

60. Kido T, Kurata A, Higashino H, et al. Cardiac imaging using 256-detector row four-dimensional CT: preliminary clinical report. Radiat Med. 2007;25: 38-44.

61. Napoli A, Fleischmann D, Chan FP, et al. Computed tomography angiography: state-of-the-art imaging using multidetector-row technology. J Comput Assist Tomogr. 2004;28(suppl 1):S32-S45.

62. Johnson TR, Krauss B, Sedlmair M, et al. Material differentiation by dual energy CT: initial experience. Eur Radiol. 2007;17:1510-1517.
63. Kinahan PE, Alessio AM, Fessler JA. Dual energy CT attenuation correction methods for quantitative assessment of response to cancer therapy with PET/ CT imaging. Technol Cancer Res Treat. 2006;5:319-327.

64. Townsend DW, Beyer T. A combined PET/CT scanner: the path to true image fusion. Br J Radiol. 2002;75 Spec No:S24-30.

65. Beyer T, Townsend DW, Blodgett TM. Dual-modality PET/CT tomography for clinical oncology. Q J Nucl Med. 2002;46:24-34.

66. Townsend DW. A combined PET/CT scanner: the choices. J Nucl Med. 2001;42:533-534.

67. Townsend DW, Beyer T, Blodgett TM. PET/CT scanners: a hardware approach to image fusion. Semin Nucl Med. 2003;33:193-204.

68. Hamoir X, Salovic D, Bouziane T, Kirsch J. Dual source CT: cardio-pulmonary applications. JBR-BTR. 2007;90:77-79.

69. Rehfeld NS, Heismann BJ, Kupferschläger J, et al. Single and dual energy attenuation correction in PET/CT in the presence of iodine based contrast agents. Phys Med Biol. 2008. In press.

70. Luker GD, Luker KE. Optical imaging: current applications and future directions. J Nucl Med. 2007;49:1-4.

71. Pablos JL, Santiago B, Galindo M, et al. Synoviocyte-derived CXCL12 is displayed on endothelium and induces angiogenesis in rheumatoid arthritis. J Immunol. 2003;170:2147-2152.

72. Paulmurugan R, Gambhir SS. Firefly luciferase enzyme fragment complementation for imaging in cells and living animals. Anal Chem. 2005;77:1295-1302.

73. Deliolanis N, Lasser T, Hyde D, Soubret A, Ripoll J, Ntziachristos V. Freespace fluorescence molecular tomography utilizing 360 degrees geometry projections. Opt Lett. 2007;32:382-384.

74. Ntziachristos V, Tung CH, Bremer C, Weissleder R. Fluorescence molecular tomography resolves protease activity in vivo. Nat Med. 2002;8:757-760.

75. Zavattini G, Vecchi S, Mitchell G, et al. A hyperspectral fluorescence system for 3D in vivo optical imaging. Phys Med Biol. 2006;51:2029-2043.

76. Cutler M. Transillumination of the breast. Ann Surg. 1931;93:223-234.

77. Ntziachristos V, Chance B. Probing physiology and molecular function using optical imaging: applications to breast cancer. Breast Cancer Res. 2001;3:41-46.

78. Schenck JF. Safety of strong, static magnetic fields. J Magn Reson Imaging. 2000;12:2-19.

79. Damadian R. Tumor detection by nuclear magnetic resonance. Science. 1971;171:1151-1153

80. Elgort DR, Duerk JL. A review of technical advances in interventional magnetic resonance imaging. Acad Radiol. 2005;12:1089-1099.

81. Alger JR, Cloughesy TF. Structural and functional imaging of cerebral neoplasia. In: Mazzuiotta JC, Toga AW, Fackowiak RSJ, eds. Brain Mapping: The Disorders. San Diego, CA: Academic Press; 2000:387-416.

82. Hendrick RE, Haacke EM. Basic physics of MR contrast agents and maximization of image contrast. J Magn Reson Imaging. 1993;3:137-148.

83. Cha S, Johnson G, Wadghiri YZ, et al. Dynamic, contrast-enhanced perfusion MRI in mouse gliomas: correlation with histopathology. Magn Reson Med. 2003;49:848-855.

84. Ostergaard L, Sorensen AG, Kwong KK, Weisskoff RM, Gyldensted C, Rosen BR. High resolution measurement of cerebral blood flow using intravascular tracer bolus passages. Part II: experimental comparison and preliminary results. Magn Reson Med. 1996;36:726-736.

85. Ostergaard L, Weisskoff RM, Chesler DA, Gyldensted C, Rosen BR. High resolution measurement of cerebral blood flow using intravascular tracer bolus passages. Part I: mathematical approach and statistical analysis. Magn Reson Med. 1996;36:715-725.

86. Frahm J, Bruhn H, Gyngell ML, Merboldt KD, Hänicke W, Sauter R. Localized high-resolution proton NMR spectroscopy using stimulated echoes: initial applications to human brain in vivo. Magn Reson Med. 1989;9:79-93.

87. Bottomley PA. Spatial localization in NMR spectroscopy in vivo. Ann N Y Acad Sci. 1987;508:333-348.

88. Ordidge RJ. Volume selection for in-vivo biological spectroscopy. In: Govil G, Khetrapal CL, Saran A, eds. Magnetic Resonance in Biology and Medicine. New Delhi, India: Tata McGraw-Hill; 1985:387-397.

89. Alger JR, Frank JA, Bizzi A, et al. Metabolism of human gliomas: assessment with H-1 MR spectroscopy and F-18 fluorodeoxyglucose PET. Radiology. 1990;177:633-641.

90. Majos C, Alonso J, Aguilera C, et al. Adult primitive neuroectodermal tumor: proton MR spectroscopic findings with possible application for differential diagnosis. Radiology. 2002;225:556-566.

91. Negendank WG, Sauter R, Brown TR, et al. Proton magnetic resonance spectroscopy in patients with glial tumors: a multicenter study. J Neurosurg. 1996;84:449-458.

92. Ott D, Hennig J, Ernst T. Human brain tumors: assessment with in vivo proton MR spectroscopy. Radiology. 1993;186:745-752. 
93. Peeling J, Sutherland G. High-resolution ${ }^{1} \mathrm{H}$ NMR spectroscopy studies of extracts of human cerebral neoplasms. Magn Reson Med. 1992;24:123-136.

94. Frahm J, Bruhn H, Hanicke W, Merboldt KD, Mursch K, Markakis E. Localized proton NMR spectroscopy of brain tumors using short-echo time STEAM sequences. J Comput Assist Tomogr. 1991;15:915-922.

95. Croteau D, Scarpace L, Hearshen D, et al. Correlation between magnetic resonance spectroscopy imaging and image-guided biopsies: semiquantitative and qualitative histopathological analyses of patients with untreated glioma. Neurosurgery. 2001;49:823-829.

96. Dowling C, Bollen AW, Noworolski SM, et al. Preoperative proton MR spectroscopic imaging of brain tumors: correlation with histopathologic analysis of resection specimens. AJNR. 2001;22:604-612.

97. Stadlbauer A, Gruber S, Nimsky C, et al. Preoperative grading of gliomas by using metabolite quantification with high-spatial-resolution proton MR spectroscopic imaging. Radiology. 2006;238:958-969.

98. Büchert M, O’Neill JPV, Maudsley AA. In vivo brain $\mathrm{pH}$ mapping using 19x19 voxels ${ }^{1} \mathrm{H}$ MR spectroscopic imaging of the down field region [abstract]. In: Proceedings of the International Society for Magnetic Resonance in Medicine: 9th Scientific Meeting and Exhibition. Berkeley, CA: International Society for Magnetic Resonance in Medicine; 2001:1701.

99. Kuroda K, Suzuki Y, Ishihara Y, Okamoto K, Suzuki Y. Temperature mapping using water proton chemical shift obtained with 3D-MRSI: feasibility in vivo. Magn Reson Med. 1996;35:20-29.

100. Richards TL, Gates GA, Gardner JC, et al. Functional MR spectroscopy of the auditory cortex in healthy subjects and patients with sudden hearing loss. AJNR Am J Neuroradiol. 1997;18:611-620.

101. Neubauer S. High-energy phosphate metabolism in normal, hypertrophied and failing human myocardium. Heart Fail Rev. 1999;4:269-280.

102. Bowers CR, Weitekamp DP. Transformation of symmetrization order to nuclear-spin magnetization by chemical reaction and nuclear magnetic resonance. Phys Rev Lett. 1986;57:2645-2648.

103. Bowers CR, Weitekamp DP. Parahydrogen and synthesis allow dramatically enhanced nuclear alignment. J Am Chem Soc. 1987;109:5541-5542.

104. Abragam A, Goldman M. Principles of dynamic nuclear polarisation. Rep Prog Phys. 1978;41:395-467.

105. Goldman M. Spin temperature and nuclear magnetic resonance in solids. Oxford, U.K.: Oxford University Press; 1970.

106. Driehuys B, Cofer GP, Pollaro J, Mackel JB, Hedlund LW, Johnson GA. Imaging alveolar-capillary gas transfer using hyperpolarized 129Xe MRI. Proc Natl Acad Sci USA. 2006;103:18278-18283.

107. van Beek EJ, Wild JM. Hyperpolarized 3-helium magnetic resonance imaging to probe lung function. Proc Am Thorac Soc. 2005;2:528-532, 510.

108. Schroder L, Lowery TJ, Hilty C, Wemmer DE, Pines A. Molecular imaging using a targeted magnetic resonance hyperpolarized biosensor. Science. 2006;314:446-449.

109. Olsson LE, Chai CM, Axelsson O, Karlsson M, Golman K, Petersson JS. MR coronary angiography in pigs with intraarterial injections of a hyperpolarized ${ }^{13}$ C substance. Magn Reson Med. 2006;55:731-737.

110. Svensson J, Mansson S, Johansson E, Petersson JS, Olsson LE. Hyperpolarized ${ }^{13} \mathrm{C}$ MR angiography using trueFISP. Magn Reson Med. 2003;50: $256-262$.

111. Golman K, Zandt RI, Lerche M, Pehrson R, Ardenkjaer-Larsen JH. Metabolic imaging by hyperpolarized ${ }^{13} \mathrm{C}$ magnetic resonance imaging for in vivo tumor diagnosis. Cancer Res. 2006;66:10855-10860.

112. Mansson S, Johansson E, Magnusson P, et al. ${ }^{13} \mathrm{C}$ imaging: a new diagnostic platform. Eur Radiol. 2006;16:57-67.

113. Bihan DL. Applications to functional MRI. In: Bihan DL, ed. Diffusion and Perfusion Magnetic Resonance Imaging. New York, NY: Raven; 1995.

114. Sorensen AG, Copen WA, Ostergaard L, et al. Hyperacute stroke: simultaneous measurement of relative cerebral blood volume, relative cerebral blood flow, and mean tissue transit time. Radiology. 1999;210:519-527.

115. Ikezaki K, Takahashi M, Koga H, et al. Apparent diffusion coefficient (ADC) and magnetization transfer contrast (MTC) mapping of experimental brain tumor. Acta Neurochir Suppl. 1997;70:170-172.

116. Provenzale JM, Mukundan S, Barboriak DP. Diffusion-weighted and perfusion MR imaging for brain tumor characterization and assessment of treatment response. Radiology. 2006;239:632-649.

117. Ono J, Harada K, Mano T, Sakurai K, Okada S. Differentiation of dys- and demyelination using diffusional anisotropy. Pediatr Neurol. 1997;16:63-66.

118. Hanyu H, Sakurai H, Iwamoto T, Takasaki M, Shindo H, Abe K. Diffusionweighted MR imaging of the hippocampus and temporal white matter in Alzheimer's disease. J Neurol Sci. 1998;156:195-200.

119. Le Bihan D. Looking into the functional architecture of the brain with diffusion MRI. Nat Rev Neurosci. 2003;4:469-480.
120. Koh DM, Collins DJ. Diffusion-weighted MRI in the body: applications and challenges in oncology. AJR. 2007;188:1622-1635.

121. Darquie A, Poline JB, Poupon C, Saint-Jalmes H, Le Bihan D. Transient decrease in water diffusion observed in human occipital cortex during visual stimulation. Proc Natl Acad Sci USA. 2001;98:9391-9395.

122. Le Bihan D, Urayama S, Aso T, Hanakawa T, Fukuyama H. Direct and fast detection of neuronal activation in the human brain with diffusion MRI. Proc Natl Acad Sci USA. 2006;103:8263-8268.

123. Ogawa S, Lee TM. Magnetic resonance imaging of blood vessels at high fields: in vivo and in vitro measurements and image simulation. Magn Reson Med. 1990;16:9-18.

124. Ogawa S, Lee TM, Kay AR, Tank DW. Brain magnetic resonance imaging with contrast dependent on blood oxygenation. Proc Natl Acad Sci USA. 1990;87: 9868-9872.

125. Ogawa S, Lee TM, Nayak AS, Glynn P. Oxygenation-sensitive contrast in magnetic resonance image of rodent brain at high magnetic fields. Magn Reson Med. 1990;14:68-78.

126. Kwong KK, Belliveau JW, Chesler DA, et al. Dynamic magnetic resonance imaging of human brain activity during primary sensory stimulation. Proc Natl Acad Sci USA. 1992;89:5675-5679.

127. Amaro E Jr, Barker GJ. Study design in fMRI: basic principles. Brain Cogn. 2006;60:220-232.

128. Bandettini PA, Cox RW. Event-related fMRI contrast when using constant interstimulus interval: theory and experiment. Magn Reson Med. 2000;43:540-548.

129. Friston KJ, Holmes AP, Worsley KJ, Poline JP, Frith CD, Frackowiak RSJ. Statistical parametric maps in functional imaging: a general linear approach. Hum Brain Mapp. 1994;2:189-210.

130. Huettel SA, Song AW, McCarthy G. Applications of fMRI. In: Huettel SA, Song AW, McCarthy G, eds. Functional Magnetic Resonance Imaging. Sunderland, U.K.: Sinauer; 2004.

131. Barrett T, Brechbiel M, Bernardo M, Choyke PL. MRI of tumor angiogenesis. J Magn Reson Imaging. 2007;26:235-249.

132. Yetkin FZ, Mendelsohn D. Hypoxia imaging in brain tumors. Neuroimaging Clin N Am. 2002;12:537-552.

133. Sunaert S. Presurgical planning for tumor resectioning. J Magn Reson Imaging. 2006;23:887-905.

134. Hoult DI, Chen CN, Sank VJ. The field dependence of NMR imaging. II. Arguments concerning an optimal field strength. Magn Reson Med. 1986;3: 730-746.

135. Schick F. Whole-body MRI at high field: technical limits and clinical potential. Eur Radiol. 2005;15:946-959.

136. Tkac I, Andersen P, Adriany G, Merkle H, Ugurbil K, Gruetter R. In vivo ${ }^{1} \mathrm{H}$ NMR spectroscopy of the human brain at 7 T. Magn Reson Med. 2001;46:451456.

137. Ugurbil K, Adriany G, Andersen P, et al. Ultrahigh field magnetic resonance imaging and spectroscopy. Magn Reson Imaging. 2003;21:1263-1281.

138. Vaughan JT, Garwood M, Collins CM, et al. 7T vs. 4T: RF power, homogeneity, and signal-to-noise comparison in head images. Magn Reson Med. 2001;46:2430 .

139. Vaughan T, DelaBarre L, Snyder C, et al. 9.4T human MRI: preliminary results. Magn Reson Med. 2006;56:1274-1282.

140. van den Bergen B, Van den Berg CA, Bartels LW, Lagendijk JJ. 7 T body MRI: B1 shimming with simultaneous SAR reduction. Phys Med Biol. 2007;52: 5429-5441.

141. Wiesinger F, Van de Moortele PF, Adriany G, De Zanche N, Ugurbil K, Pruessmann KP. Potential and feasibility of parallel MRI at high field. NMR Biomed. 2006;19:368-378.

142. Doty FD, Entzminger G, Kulkarni J, Pamarthy K, Staab JP. Radio frequency coil technology for small-animal MRI. NMR Biomed. 2007;20:304-325.

143. Griswold MA, Jakob PM, Heidemann RM, et al. Generalized autocalibrating partially parallel acquisitions (GRAPPA). Magn Reson Med. 2002;47:1202-1210.

144. Pruessmann KP, Weiger M, Scheidegger MB, Boesiger P. SENSE: sensitivity encoding for fast MRI. Magn Reson Med. 1999;42:952-962.

145. Schmidt GP, Baur-Melnyk A, Herzog P, et al. High-resolution whole-body magnetic resonance image tumor staging with the use of parallel imaging versus dual-modality positron emission tomography-computed tomography: experience on a 32-channel system. Invest Radiol. 2005;40:743-753.

146. Schaefer JF, Schlemmer HP. Total-body MR-imaging in oncology. Eur Radiol. 2006;16:2000-2015.

147. Sommer G, Fautz HP, Ludwig U, Hennig J. Multicontrast sequences with continuous table motion: a novel acquisition technique for extended field of view imaging. Magn Reson Med. 2006;55:918-922.

148. Beyer T, Townsend DW, Brun T, et al. A combined PET/CT scanner for clinical oncology. J Nucl Med. 2000;41:1369-1379. 
149. Nakamoto Y, Chin BB, Cohade C, Osman M, Tatsumi M, Wahl RL. PET/CT: artifacts caused by bowel motion. Nucl Med Commun. 2004;25:221-225.

150. Osman MM, Cohade C, Nakamoto Y, Marshall LT, Leal JP, Wahl RL. Clinically significant inaccurate localization of lesions with PET/CT: frequency in 300 patients. $J$ Nucl Med. 2003;44:240-243.

151. Osman MM, Cohade C, Nakamoto Y, Wahl RL. Respiratory motion artifacts on PET emission images obtained using CT attenuation correction on PET-CT. Eur J Nucl Med Mol Imaging. 2003;30:603-606.

152. Goerres GW, Kamel E, Seifert B, et al. Accuracy of image coregistration of pulmonary lesions in patients with non-small cell lung cancer using an integrated PET/CT system. J Nucl Med. 2002;43:1469-1475.

153. Alavi A, Mavi A, Basu S, Fischman A. Is PET-CT the only option? Eur J Nucl Med Mol Imaging. 2007;34:819-821.

154. Shao Y, Cherry SR, Farahani K, et al. Development of a PET detector system compatible with MRI/NMR systems. IEEE Trans Nucl Sci. 1997;44:1167-1171.

155. Marsden PK, Strul D, Keevil SF, Williams SC, Cash D. Simultaneous PET and NMR. Br J Radiol. 2002;75(spec no.):S53-S59.

156. Raylman RR, Majewski S, Lemieux SK, et al. Simultaneous MRI and PET imaging of a rat brain. Phys Med Biol. 2006;51:6371-6379.

157. Lucas AJ, Hawkes RC, Ansorge RE, et al. Development of a combined microPET-MR system. Technol Cancer Res Treat. 2006;5:337-341.

158. Gilbert KM, Handler WB, Scholl TJ, Odegaard JW, Chronik BA. Design of field-cycled magnetic resonance systems for small animal imaging. Phys Med Biol. 2006;51:2825-2841.

159. Handler WB, Gilbert KM, Peng H, Chronik BA. Simulation of scattering and attenuation of $511 \mathrm{keV}$ photons in a combined PET/field-cycled MRI system. Phys Med Biol. 2006;51:2479-2491.

160. Schlemmer H-P, Pichler BJ, Wienhard K, et al. Simultaneous MR/PET for brain imaging: first patient scans [abstract]. J Nucl Med. 2007;48(suppl 2):45P.
161. Schmand M, Burbar Z, Corbeil JL, et al. Brain PET: First human tomograph for simultaneous (functional) PET and MR imaging [abstract]. J Nucl Med. 2007;48(suppl):45P.

162. Zaidi H. Is MR-guided attenuation correction a viable option for dual-modality PET/MR imaging? Radiology. 2007;244:639-642.

163. Hofmann M, Steinke F, Scheel V, et al. MR-based PET attenuation correction: method and validation. In: 2007 IEEE Nuclear Science Symposium Conference Record. Piscataway, NJ: IEEE; 2007.

164. Hammer BE, Christensen NL, Heil BG. Use of a magnetic field to increase the spatial resolution of positron emission tomography. Med Phys. 1994;21:1917-1920.

165. Meltzer CC, Leal JP, Mayberg HS, Wagner HN Jr, Frost JJ. Correction of PET data for partial volume effects in human cerebral cortex by MR imaging. J Comput Assist Tomogr. 1990;14:561-570.

166. Rousset OG, Ma Y, Evans AC. Correction for partial volume effects in PET: principle and validation. J Nucl Med. 1998;39:904-911.

167. Garlick PB, Marsden PK, Cave AC, et al. PET and NMR dual acquisition (PANDA): applications to isolated, perfused rat hearts. NMR Biomed. 1997;10:138-142.

168. Bailey DL, Townsend DW, Valk PE, Maisey MN. Positron Emission Tomography. New York, NY: Springer; 2005:29-34.

169. van Eijk CW. Inorganic scintillators in medical imaging. Phys Med Biol. 2002;47:R85-R106

170. Pepin CM, Berard P, Perrot AL, et al. Properties of LYSO and recent LSO scintillators for phoswich PET detectors. IEEE Trans Nucl Sci. 2004;51:789.

171. Phelps ME, Hoffman EJ, Mullani NA, Ter-Pogossian MM. Application of annihilation coincidence detection to transaxial reconstruction tomography. J Nucl Med. 1975;16:210-224.

172. Schmand M, Eriksson C, Casey ME, et al. Performance results of a new DOI detector block for a high resolution PET-LSO research tomograph HRRT. IEEE Trans Nucl Sci. 1998;45:3000-3006. 